\title{
On the $\alpha$-element gradients of the Galactic thin disk using Cepheids ${ }^{\star} \star \star$
}

\author{
K. Genovali ${ }^{1}$, B. Lemasle ${ }^{2}$, R. da Silva ${ }^{1}$, G. Bono ${ }^{1,3}$, M. Fabrizio ${ }^{4}$, M. Bergemann ${ }^{5,6}$, \\ R. Buonanno ${ }^{1,4}$, I. Ferraro ${ }^{3}$, P. François ${ }^{7,8}$, G. Iannicola ${ }^{3}$, L. Inno ${ }^{1,9}$, C. D. Laney ${ }^{10,11}$, R.-P. Kudritzki ${ }^{12,13,14}$, \\ N. Matsunaga ${ }^{15}$, M. Nonino ${ }^{16}$, F. Primas ${ }^{9}$, M. Romaniello ${ }^{9}$, M. A. Urbaneja ${ }^{17}$, and F. Thévenin ${ }^{18}$ \\ ${ }^{1}$ Dipartimento di Fisica, Università di Roma Tor Vergata, via della Ricerca Scientifica 1, 00133 Rome, Italy \\ e-mail: ron.oliveirasilva@gmail.com \\ 2 Anton Pannekoek Institute for Astronomy, University of Amsterdam, Science Park 904, PO Box 94249, 1090 GE Amsterdam, \\ The Netherlands \\ 3 INAF-Osservatorio Astronomico di Roma, via Frascati 33, Monte Porzio Catone, 00136 Rome, Italy \\ 4 INAF-Osservatorio Astronomico di Teramo, via Mentore Maggini s.n.c., 64100 Teramo, Italy \\ Max-Planck-Institut für Astronomy, 69117 Heidelberg, Germany \\ 6 Institute of Astronomy, University of Cambridge, Madingley Road, CB3 0HA, Cambridge, UK \\ 7 GEPI, Observatoire de Paris, CNRS, Université Paris Diderot, Place Jules Janssen, 92190 Meudon, France \\ ${ }^{8}$ UPJV, Université de Picardie Jules Verne, 33 rue St. Leu, 80080 Amiens, France \\ 9 European Southern Observatory, Karl-Schwarzschild-Str. 2, 85748 Garching bei München, Germany \\ 10 Department of Physics and Astronomy, N283 ESC, Brigham Young University, Provo, UT 84601, USA \\ 11 South African Astronomical Observatory, PO Box 9, Observatory 7935, South Africa \\ 12 Institute for Astronomy, University of Hawaii, 2680 Woodlawn Drive, Honolulu, HI 96822, USA \\ 13 Max-Planck-Institute for Astrophysics, Karl-Schwarzschild-Str.1, 85741 Garching, Germany \\ ${ }^{14}$ University Observatory Munich, Scheinerstr. 1, 81679 Munich, Germany \\ 15 Department of Astronomy, School of Science, The University of Tokyo, 7-3-1 Hongo, Bunkyo-ku, 113-0033 Tokyo, Japan \\ 16 INAF-Osservatorio Astronomico di Trieste, via G. B. Tiepolo 11, 34143 Trieste, Italy \\ 17 Institute for Astro- and Particle Physics, University of Innsbruck, Technikerstr. 25/8, 6020 Innsbruck, Austria \\ 18 Laboratoire Lagrange, CNRS/UMR 7293, Observatoire de la Côte d'Azur, Bd de l'Observatoire, CS 34229, 06304 Nice, France
}

Received 13 February 2015 / Accepted 11 March 2015

\begin{abstract}
We present new homogeneous measurements of $\mathrm{Na}, \mathrm{Al}$, and three $\alpha$-elements $(\mathrm{Mg}, \mathrm{Si}, \mathrm{Ca})$ for $75 \mathrm{Galactic}$ Cepheids. The abundances are based on high spectral resolution $(R \sim 38000)$ and high signal-to-noise ratio $(S / N \sim 50-300)$ spectra collected with UVES at ESO VLT. The current measurements were complemented with Cepheid abundances provided by our group (75) or available in the literature, for a total of 439 Galactic Cepheids. Special attention was given to providing a homogeneous abundance scale for these five elements plus iron. In addition, accurate Galactocentric distances $\left(R_{\mathrm{G}}\right)$ based on near-infrared photometry are also available for all the Cepheids in the sample. They cover a large section of the Galactic thin disk $\left(4.1 \leq R_{\mathrm{G}} \leq 18.4 \mathrm{kpc}\right)$. We found that these five elements display well-defined linear radial gradients and modest standard deviations over the entire range of $R_{\mathrm{G}}$. Moreover, the [element/Fe] abundance ratios are constant across the entire thin disk; only the Ca radial distribution shows marginal evidence of a positive slope. These results indicate that the chemical enrichment history of iron and of the quoted five elements has been quite similar across the four quadrants of the Galactic thin disk. The [element/Fe] ratios are also constant over the entire period range. This empirical evidence indicates that the chemical enrichment of Galactic Cepheids has also been very homogenous within the range in age that they cover ( 10-300 Myr). Once again, $[\mathrm{Ca} / \mathrm{Fe}]$ vs. $\log P$ shows a (negative) gradient, since it is underabundant among the youngest Cepheids. Finally, we also find that Cepheid abundances agree quite well with similar abundances for thin and thick disk dwarf stars, and they follow the typical $\mathrm{Mg}-\mathrm{Al}$ and $\mathrm{Na}-\mathrm{O}$ correlations.
\end{abstract}

Key words. stars: abundances - stars: variables: Cepheids - stars: oscillations - Galaxy: disk - open clusters and associations: general

\section{Introduction}

Massive and intermediate-mass stars play a crucial role in different fields of modern astrophysics. They are the main contributors

* Based on spectra collected with the UVES spectrograph available at the ESO Very Large Telescope (VLT), Cerro Paranal, Chile (ESO Proposals: 081.D-0928(A), PI: S. Pedicelli; 082.D-0901(A), PI: S. Pedicelli; 089.D-0767(C), PI: K. Genovali).

$\star \star$ Tables 1,2 , and 5 are only available at the CDS via anonymous ftp to cdsarc.u-strasbg.fr $(130.79 .128 .5)$ or via

http://cdsarc.u-strasbg.fr/viz-bin/qcat?]/A+A/580/A17 in the UV and in the NIR emission of unresolved stellar systems (Crowther 2007). Moreover, they also play a crucial role in the chemical enrichment of the interstellar medium (Ventura et al. 2002; Decressin et al. 2007; Maeder 2009; Karakas et al. 2012). Blue and red supergiants are also fundamental stellar tracers for constraining the metallicity gradients in external early-type galaxies (Urbaneja et al. 2005; Kudritzki et al. 2008; Bresolin 2011; Evans et al. 2011).

The distribution of the elements throughout the disk of external spiral galaxies generally follows a radial gradient with metallicity increasing towards the centre. This can be seen, for 
instance, in M 31 (e.g., Sanders et al. 2012; Zurita \& Bresolin 2012), M 33 (Magrini et al. 2010a), M 81 (e.g., Kudritzki et al. 2012; Stanghellini et al. 2014), NGC 300 (e.g., Stasinka et al. 2013), NGC 3621 (e.g., Kudritzki et al. 2014), NGC 5668 (e.g., Marino et al. 2012), or in large samples, such as those of Pilyugin et al. (2014a) and Sanchez et al. (2014). The Milky Way shows an iron gradient comparable to other galaxies, in particular a well-investigated negative iron gradient (see, e.g., Luck \& Lambert 2011; Genovali et al. 2013, and references therein). In general, shallower $[\mathrm{O} / \mathrm{H}]$ gradients (Vila-Costas \& Edmunds 1992; Edmunds \& Roy 1993; Zaritsky et al. 1994; Martin \& Roy 1994) have been found in barred galaxies in comparison with the ones in unbarred galaxies (but see also Pilyugin et al. 2014b; Sanchez et al. 2014).

The gradient in the Milky Way has been investigated well using different stellar tracers of different ages and natures: H II regions, O/B-type stars, Cepheids, open clusters (OC), and planetary nebulae $(\mathrm{PNe})$. Along with the iron gradient, several authors have investigated the $\alpha$-element abundances throughout the disk (e.g., Yong et al. 2006; Lemasle et al. 2007, 2013; Luck et al. 2011; Luck \& Lambert 2011) and provided gradients based on Cepheids spanning from -0.039 to $-0.095 \mathrm{dex} \mathrm{kpc}^{-1}$, depending on the element. In particular, Korotin et al. (2014) used non-LTE abundance analysis of the oxygen triplet in the near-infrared to give an $[\mathrm{O} / \mathrm{H}]$ gradient of $-0.058 \mathrm{dex} \mathrm{kpc}^{-1}$.

$[\mathrm{O} / \mathrm{H}]$ gradients spanning -0.030 to $-0.08 \mathrm{dex} \mathrm{kpc}^{-1}$ have been inferred from H II regions (e.g., Quireza et al. 2006; Rudolph et al. 2006; Balser et al. 2011), which is very similar to what has been obtained from O-B1 stars, -0.03 and $-0.07 \mathrm{dex} \mathrm{kpc}^{-1}$ (see, e.g., Smartt \& Rolleston 1997; Daflon \& Cunha 2004; Rolleston et al. 2000). In particular, Daflon \& Cunha (2004) provide abundance gradients based on $\alpha$ elements in OB stars spanning from $-0.032 \pm 0.012 \mathrm{dex} \mathrm{kpc}^{-1}$ for oxygen to $-0.052 \pm 0.014 \mathrm{dex} \mathrm{kpc}^{-1}$ for magnesium.

Planetary nebulae observed up to Galactocentric distances $\left(R_{\mathrm{G}}\right)=10 \mathrm{kpc}$ show either shallow $[\mathrm{O} / \mathrm{H}]$ gradients from -0.02 to $-0.085 \mathrm{dex} \mathrm{kpc}^{-1}$ (Maciel \& Quireza 1999; Costa et al. 2004; Henry et al. 2004, 2010; Perinotto \& Morbidelli 2006; Pottasch \& Bernard-Salas 2006) or no evidence of a radial gradient (Stanghellini et al. 2006).

Twarog et al. (1997) first suggested that instead of a break in the slope, the radial metallicity gradient could experience an abrupt drop of $0.2-0.3 \mathrm{dex}$ around $10 \mathrm{kpc}$. This feature has also been proposed in other studies (e.g., Daflon \& Cunha 2004; Andrievsky et al. 2004). The exact location of the possible jump in metallicity has not been properly defined. Lépine et al. (2011) claim that there is such a metallicity discontinuity in the gradients of both iron and $\alpha$ elements (an average of $\mathrm{O}, \mathrm{Si}, \mathrm{S}, \mathrm{Mg}$, and $\mathrm{Ca}$ ) based on Cepheids, and they associate it with the depression in velocity displayed around $10 \mathrm{kpc}$ in the rotation curve of the Galaxy.

The theoretical scenario that seems to describe the gradient of iron and $\alpha$ elements in the Milky Way better is the so-called inside-out scenario (e.g., Matteucci \& François 1989; Chiappini et al. 1997, 2001; Prantzos \& Boissier 2000; Mollá \& Díaz 2005; Fu et al. 2009). According to this model, the Milky Way forms primarily during two episodes of gas infall (the first giving birth to the halo and the bulge, the second producing the disk) with an almost independent evolution between the halo and the thin disk.

Although the inside-out model is not unique, it reproduces the majority of the observed features of the Milky Way. Cescutti et al. (2007) computed new radial gradients for numerous elements and closely reproduced Cepheid-based observations based on the nucleosynthesis prescription provided by François et al. (2004), who adopted the star formation and infall laws based on observed features of the Milky Way. The most important factor in reproducing the $[$ element $/ \mathrm{Fe}] \mathrm{vs} .[\mathrm{Fe} / \mathrm{H}]$ relations, as well as the solar absolute abundances in the solar neighborhood, is the combination of the yields from supernovae ( $\mathrm{SNe}$ ) Types Ia and II, whose progenitors are, respectively, low/intermediate mass and massive stars. In this context, it is worth mentioning that $\alpha$-element abundances also play a crucial role in constraining the plausibility of the physical assumptions (instantaneous mixing, instantaneous recycling) adopted to compute chemical evolution models (Spitoni et al. 2013a).

The $\alpha$-enrichment of Cepheids is also often investigated by means of abundance gradients scaled to iron. Iron is mainly produced in $\mathrm{SNe}$ Ia, with contributions from SNe II, whereas $\alpha$-elements are the principal yields of core-collapse $\mathrm{SNe}$, but with contributions of $\mathrm{SNe}$ Ia for $\mathrm{Ca}$ and $\mathrm{Si}$. Therefore, the $[\alpha$-element $/ \mathrm{Fe}]$ ratio is an interesting diagnostic for constraining the chemical enrichment history. A comprehensive study of open-cluster abundance gradients was provided by Yong et al. (2012) after collecting results from different groups (Bragaglia et al. 2008; Carraro et al. 2007a,b; Chen et al. 2003; Friel et al. 2002, 2010; Jacobson et al. 2008, 2009, 2011a,b; Magrini et al. 2009, 2010b; Pancino et al. 2010; Sestito et al. 2006, 2007; Yong et al. 2005). They find different trends for the $[\alpha / \mathrm{Fe}]$ ratios based on open clusters. $\mathrm{Ca}$ abundances attain solar values over the entire range of Galactocentric distances, while $\mathrm{Mg}$ and Si display marginal evidence of a slope $\left(\sim 0.01-0.02 \mathrm{dex} \mathrm{kpc}^{-1}\right)$. On the other hand, the average $[\alpha / \mathrm{Fe}]$ ratio attains enhanced values in the outer disk (see their Fig. 21) thanks to the increase in $\mathrm{O}$ and $\mathrm{Ti}$ abundances. However, as noted by the anonymous referee, $\mathrm{Ti}$ abundances display large variations in different investigations and may be dominated by systematic effects. In general, the large majority of investigations of radial dependence based on OCs have found a linear gradient of approximately $-0.06 \mathrm{dex} \mathrm{kpc}^{-1}$ in the range of $5-10 \mathrm{kpc}$. Concerning the outer disk, however, several authors find evidence of a flattening of the gradients $([\mathrm{Fe} / \mathrm{H}] \sim-0.3)$ for distances greater than $12-14 \mathrm{kpc}$ (Magrini et al. 2009; Pancino et al. 2010; Yong et al. 2012, and references therein).

The abundance gradients of the elements in the disk of the Milky Way provide strong constraints for chemical evolution models because they are connected to the evolution of the Galaxy disk. In this context, the $\alpha$ elements play a key role since they are good tracers of the chemical enrichment history of stellar populations. In particular, an overabundance of $\alpha$ elements is typically associated with a fast chemical enrichment in which iron played a minor role (Tinsley 1979; Matteucci 2003). However, the theoretical and empirical scenario is far from being settled, since the initial mass function (IMF, Calura et al. 2010) and the radial gas flows (Spitoni et al. 2013b) can also affect the abundance gradients. In particular, gas flows can have similar effects on the gas density distribution, and in turn they can have an impact on the star formation rate (Colavitti et al. 2008, and references therein). Recent chemical evolution models, including both radial gas flows and radial stellar migrations (Kubryk et al. 2015a,b; Minchev et al. 2013, 2014), indicate a steady decrease in the slope as a function of time.

In this paper we investigate the gradient associated to three $\alpha$ elements $(\mathrm{Mg}, \mathrm{Si}, \mathrm{Ca})$, together with $\mathrm{Na}$ and $\mathrm{Al}$, focusing on the inner and outer disk regions. The paper is organized as follows. In Sect. 2 we briefly recall the observations and data analysis. In Sect. 3 we present and discuss the radial gradients, and in Sect. 4 the metallicity distributions. In Sect. 5 we discuss 
some correlations involving the derived abundances, and finally, in Sect. 6 we give a summary of our findings.

\section{Observations, data reduction, and analysis}

\subsection{Spectroscopic data}

In this work we used the same high-resolution $(R \sim 38000)$ and high signal-to-noise ratio $(\mathrm{S} / \mathrm{N})$ spectra as were analyzed by Genovali et al. (2014, hereafter G14). The spectra of 75 Galactic Cepheids were collected with the UVES spectrograph at the ESO VLT (Cerro Paranal, Chile). In particular, we collected 122 spectra using two different instrument settings: i) the former makes use of the UVES DIC 2 configuration that allows the blue and red arms to operate in parallel. With this setting, we collected (between October 2008 and April 2009') 80 spectra of 74 stars, with wavelength ranges of $\sim 3760-4985 \AA$,

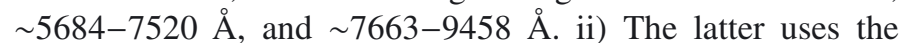
UVES red arm configuration and the cross disperser CD 3 . By adopting this setting, we collected (between May and September $2012^{2}$ ) 42 spectra of a control sample of 11 Cepheids with wavelength ranges of $\sim 4786-5750 \AA$ and $\sim 5833-6806 \AA$.

The 11 Cepheids (V340 Ara, AV Sgr, VY Sgr, UZ Sct, Z Sct, V367 Sct, WZ Sgr, XX Sgr, KQ Sco, RY Sco, V500 Sco) were used as a control sample since we had collected from four to six spectra each and with both the instrumental configurations (with the exception of V500 Sco, which has 4 spectra collected only with the second instrument setting). The $\mathrm{S} / \mathrm{N}$ are typically better than $\sim 100$ for all the échelle orders in the case of the first instrumental configuration (see examples in Fig. 1) and range from $\sim 50$ to roughly 300 for the second one. All the spectra were reduced using the ESO UVES pipeline Reflex v2.1 (Freudling et al. 2013).

The stars BB Gem and GQ Ori were initially present in the sample of G14. BB Gem still has an uncertain status since it was classified either as a classical or as a type-II Cepheid in different catalogs (see, e.g., Harris 1985; Loomis et al. 1988; Bersier et al. 1997). It has been excluded from the current investigation because its uncertain classification could lead to a wrong distance determination or to a wrong interpretation of the abundance pattern. The spectrum of GQ Ori has low $\mathrm{S} / \mathrm{N}$ and it is not good enough to provide a reliable abundance determination, so it was also excluded.

\subsection{Atmospheric parameters and abundances}

We adopted the same iron abundances and atmospheric parameters as derived by G14. The iron abundances are based on the equivalent widths (EW) of about 100-200 Fe I and about 20-40 Fe II lines, where the number of lines depends on the spectral range used. The number of lines also varies according to the metallicity and to the spectral type of the star. To determine the atmospheric parameters, we set a limit of $E W<120 \mathrm{~m} \AA$ in order to remain in the linear part of the curve of growth. For the objects where there were not enough weak lines, we increased the limit to $180 \mathrm{~m} \AA$. This slightly increases the uncertainties affecting the correlated atmospheric parameters, namely the effective temperature and the microturbulent velocity. For more details on the impact that typical uncertainties on effective temperature, surface gravity, and microturbulent velocity have on the iron abundances, see Table 2 of G14.

\footnotetext{
081.D-0928(A) and 082.D-0901(A), PI: S. Pedicelli.

2 089.D-0767(C), PI: K. Genovali.
}

The effective temperature $\left(T_{\text {eff }}\right)$ of individual spectra was estimated using the line depth ratio (LDR) method (Kovtyukh \& Gorlova 2000). The estimated values of $T_{\text {eff }}$ were validated to make certain that the Fe Iabundances do not depend on the excitation potential $\left(\chi_{\mathrm{ex}}\right)$, i.e., the slope of $[\mathrm{Fe} / \mathrm{H}]$ vs. $\chi_{\mathrm{ex}}$ should be as close to zero. The surface gravity $(\log g)$ was derived through the ionization equilibrium between Fe I and Fe II lines, and the microturbulent velocity $\left(v_{t}\right)$ by minimizing the slope in the $[\mathrm{Fe} \mathrm{I} / \mathrm{H}]$ vs. EW plot.

Concerning the $\mathrm{Na}, \mathrm{Al}$, and $\alpha(\mathrm{Mg}, \mathrm{Si}, \mathrm{Ca})$ elements, we used the linelist provided by Lemasle et al. (2013), with the same atomic parameters $\left(\chi_{\text {ex }}\right.$ and $\left.\log g f\right)$ listed in their Table A.1, but with small differences in the number of lines. We used the six Al I lines, two lines for $\mathrm{NaI}$ instead of three (the line at $5688.21 \AA$ is often too strong), the nine Ca I lines (the Ca II line was not included), and $14 \mathrm{Si}$ I lines (5665.56 $\AA$ was included but used only for the P89 stars). For magnesium, we preferred to adopt only the abundances provided by the line $\mathrm{Mg} \mathrm{I}$ at $5711.09 \AA$ because those at 8712.69 and 8736.02 $\AA$ are not available in the wavelength range of the 11 control sample stars. The line at $5711.09 \AA$ is affected by non-LTE effects. However, Merle et al. (2011) find that the non-LTE correction to the EWs is smaller than $10 \%$ in the quoted stellar parameter regime.

To minimize any systematic bias in the continuum estimate due to the subjectivity of the operator, three of us have independently performed EW measurements on a sample of selected lines. The EWs for several elements were also measured using the Automatic Routine for line equivalent widths in stellar Spectra (ARES, Sousa et al. 2007), and double-checked using the splot task of $\mathrm{IRAF}^{3}$. The internal dispersion is smaller than $6 \mathrm{~m} \AA$ and there is no evidence of systematics.

The abundances were derived with calrai, a spectrum synthesis code originally developed by Spite (1967) and regularly improved since then. The code computes synthetic spectra over a large grid of stellar atmospheres in which we can interpolate by using $1 \mathrm{D}$, hydrostatic, plane-parallel, and spherical LTE model atmospheres (MARCS, Gustafsson et al. 2008). For all the elements studied here, we assumed the standard solar abundances provided by Grevesse et al. (1996): $A(\mathrm{Fe})_{\odot}=7.50$, $A(\mathrm{Na})_{\odot}=6.33, A(\mathrm{Al})_{\odot}=6.47, A(\mathrm{Mg})_{\odot}=7.58, A(\mathrm{Si})_{\odot}=7.55$, and $A(\mathrm{Ca})_{\odot}=6.36$.

Table 1 lists the abundances obtained from individual spectra. Columns 3 and 4 show the iron abundances and the number of Fe I and Fe II lines used by G14. The other columns show our results for the abundances of $\mathrm{Na}, \mathrm{Al}$, and $\alpha$ elements, together with the number of lines used. In Table 2 we list the mean abundances computed for the stars with multiple spectra.

\subsection{Data available in the literature}

We compared our abundance estimates with the results provided by similar studies available in the literature: Lemasle et al. (2013, hereafter LEM), Luck et al. (2011, LII), Luck \& Lambert (2011, LIII), and Yong et al. (2006, YON). By comparing the stars in common among the different data sets, we evaluated the systematic difference among them. The mean differences between our measurements and those of LEM, LII, and LIII are quite small, about 0.13 dex in modulus or smaller for all the elements investigated here. The difference for $\mathrm{Mg}$ is on average 0.23 dex more abundant in our study than in LII's. The differences are larger

\footnotetext{
Image Reduction and Analysis Facility, distributed by the National Optical Astronomy Observatories (NOAO), USA.
} 
in the comparison with YON's measurements, which have abundances systematically smaller than ours and range from 0.18 dex for $\mathrm{Mg}$ up to 0.31 dex for Fe. The details on these comparisons are listed in Table 3, where we show the zero-point differences obtained by G14 for the iron abundance ratios, together with our determinations for the other elements. Each pair of data sets was chosen to maximize the number of stars in common between them. To provide a homogeneous abundance scale for Galactic Cepheids, we applied these zero-point differences to the quoted data sets, putting them on the same scale as for our current sample. The element abundances available in the literature and the rescaled values are listed in Cols. 2 to 15 of Table 5 .

The priority in using the abundances from the literature follows the same approach as adopted by G14: we first adopt the abundances provided by our group, i.e., this study and the results from LEM, and finally those provided by the other studies, namely LIII, LII, and YON in this order. We ended up with a sample of 439 Cepheids, with a homogeneous abundance scale for $\mathrm{Fe}, \mathrm{Na}, \mathrm{Al}, \mathrm{Mg}, \mathrm{Si}$, and $\mathrm{Ca}$.

\section{3. $\alpha$-element gradients}

In this section we investigate the radial gradients of $\mathrm{Na}, \mathrm{Al}$, and three $\alpha$ elements $(\mathrm{Mg}, \mathrm{Ca}, \mathrm{Si})$ across the Galactic disk using our sample of 75 classical Cepheids plus a sample of 364 Cepheids available in the literature. The iron gradient for our sample was provided by G14, together with a homogenous iron scale for the remaining objects. The current approach when compared with similar investigations available in the literature has two indisputable advantages: i) we use accurate elemental abundances based on high-resolution, high S/N spectra. Moreover, the approach to constraining the intrinsic parameters (surface gravity, effective temperature, microturbulent velocity) is identical, and we also use similar line lists. ii) The individual Cepheid distances were estimated using the same near-infrared PeriodWesenheit relations. The key advantages of distances based on this diagnostic is that they are independent of uncertainties affecting reddening corrections and minimally affected by the metallicity dependence (Inno et al. 2013).

Figure 2 shows the abundances scaled to hydrogen of $\mathrm{Na}$ and $\mathrm{Al}$ and of the three $\alpha$ elements as a function of $R_{\mathrm{G}}$ for the final sample. Objects plotted in this figure include the current 75 Cepheids plus 38 Cepheids from LEM, 263 from LIII, 61 from LII, and two from YON.

The individual Galactocentric distances of the Cepheids in the total sample have been estimated by G14. They adopt the near-infrared period-Wesenheit relations derived by Inno et al. (2013) and assume a solar Galactocentric distance of $7.94 \pm$ $0.37 \pm 0.26 \mathrm{kpc}$ (see Groenewegen et al. 2008; Matsunaga et al. 2013, and references therein). The $R_{\mathrm{G}}$ values are listed in our Table 2 and in their Table 4 . The typical final uncertainty on the distances is $\sim 5 \%$ and is mainly due to the period-Wesenheit zero-point calibration (for more details see Inno et al. 2013).

The abundances from the literature plotted in Fig. 2 were corrected by adopting the zero-point differences listed in Table 3. This figure also shows the linear least squares fits to the current sample of 75 Cepheids and to the entire sample (439). A biweight procedure (Beers et al. 1990) was adopted to remove outliers from the data fitted. The slopes and the zero points of these two radial gradients are labeled in the panels. The slopes and the zero points of the fits based on the entire sample, along with their uncertainties, and standard deviations are listed in Cols. 2 to 4 of Table 4.
Table 3. Abundance difference of stars in common among the current sample and other data sets.

\begin{tabular}{ccrc}
\hline \hline $\begin{array}{c}\text { Abundance } \\
\text { ratio }\end{array}$ & Data sets $^{a}$ & \multicolumn{1}{c}{$\begin{array}{c}\text { Zero-point } \\
\text { difference }\end{array}$} & $N_{\text {Common }}$ \\
\hline$[\mathrm{Fe} / \mathrm{H}]$ & LII-G14 & $-0.05 \pm 0.11$ & 45 \\
{$[\mathrm{Fe} / \mathrm{H}]$} & LIII-G14 & $0.03 \pm 0.08$ & 33 \\
{$[\mathrm{Fe} / \mathrm{H}]$} & LII-LEM & $0.08 \pm 0.12$ & 51 \\
{$[\mathrm{Fe} / \mathrm{H}]$} & LIII-YON & $0.34 \pm 0.20$ & 20 \\
{$[\mathrm{Na} / \mathrm{H}]$} & LII-TS & $-0.13 \pm 0.14$ & 38 \\
{$[\mathrm{Na} / \mathrm{H}]$} & LIII-TS & $-0.10 \pm 0.13$ & 34 \\
{$[\mathrm{Na} / \mathrm{H}]$} & LII-LEM & $-0.11 \pm 0.17$ & 36 \\
{$[\mathrm{Al} / \mathrm{H}]$} & LII-TS & $-0.08 \pm 0.16$ & 36 \\
{$[\mathrm{Al} / \mathrm{H}]$} & LIII-TS & $-0.03 \pm 0.14$ & 33 \\
{$[\mathrm{Al} / \mathrm{H}]$} & LII-LEM & $-0.03 \pm 0.16$ & 41 \\
{$[\mathrm{Mg} / \mathrm{H}]$} & LII-TS & $-0.23 \pm 0.24$ & 26 \\
{$[\mathrm{Mg} / \mathrm{H}]$} & LIII-TS & $-0.10 \pm 0.17$ & 30 \\
{$[\mathrm{Mg} / \mathrm{H}]$} & LII-LEM & $-0.27 \pm 0.24$ & 35 \\
{$[\mathrm{Mg} / \mathrm{H}]$} & LIII-YON & $0.08 \pm 0.15$ & 16 \\
{$[\mathrm{Si} / \mathrm{H}]$} & LII-TS & $-0.11 \pm 0.12$ & 41 \\
{$[\mathrm{Si} / \mathrm{H}]$} & LIII-TS & $-0.06 \pm 0.11$ & 33 \\
{$[\mathrm{Si} / \mathrm{H}]$} & LII-LEM & $-0.06 \pm 0.11$ & 55 \\
{$[\mathrm{Si} / \mathrm{H}]$} & LIII-YON & $0.12 \pm 0.08$ & 18 \\
{$[\mathrm{Ca} / \mathrm{H}]$} & LII-TS & $-0.11 \pm 0.17$ & 42 \\
{$[\mathrm{Ca} / \mathrm{H}]$} & LIII-TS & $-0.08 \pm 0.15$ & 32 \\
{$[\mathrm{Ca} / \mathrm{H}]$} & LII-LEM & $-0.06 \pm 0.17$ & 54 \\
{$[\mathrm{Ca} / \mathrm{H}]$} & LIII-YON & $0.14 \pm 0.11$ & 19 \\
\hline & & &
\end{tabular}

Notes. ${ }^{(a)}$ G14: Genovali et al. (2014); TS: this study; LII: Luck et al. (2011); LIII: Luck \& Lambert (2011); LEM: Lemasle et al. (2013); YON: Yong et al. (2006). The quoted errors represent the dispersion around the mean.

A glance at the data plotted in this figure shows that the occurrence of a radial gradient is definite for the five investigated elements. The main difference is that the standard deviations scale with the number of lines adopted to estimate the abundances. Indeed, the dispersion of the $\mathrm{Mg}$ gradient, based on a single line, is almost a factor of two larger than the dispersion of the Si gradient, based on 14 lines. The radial gradients of the five investigated elements attain, within the errors, similar slopes. This finding supports similar results by Lemasle et al. $(2007,2013)$ and by LII+LIII. The above result becomes even more compelling if we consider the similarity with the iron radial gradient $\left(-0.060 \pm 0.002 \mathrm{dex} \mathrm{kpc}^{-1}\right)$ shown by the same Cepheids (G14). The abundances of $\mathrm{Na}$ and $\mathrm{Al}$ and of the three $\alpha$-elements seem to show a flattening for distances greater than $\sim 13 \mathrm{kpc}$. Thus supporting a similar trend to the one found using open clusters (Yong et al. 2012). However, firm conclusions are hampered by the increased spread in abundance and by the paucity of the sample in the outermost disk regions.

The current slopes also agree, within $1 \sigma$, with similar estimates available in the literature. In our results the slopes range from $-0.055 \pm 0.003$ for $[\mathrm{Al} / \mathrm{H}]$ to $-0.039 \pm 0.002 \mathrm{dex} \mathrm{kpc}^{-1}$ for $[\mathrm{Ca} / \mathrm{H}]$. The slopes estimated by LII+LIII for the same elements range from $-0.053 \pm 0.004$ to $-0.040 \pm 0.004 \mathrm{dex} \mathrm{kpc}^{-1}$, while those estimated by LEM range from $-0.046 \pm 0.013$ to $-0.044 \pm 0.012 \mathrm{dex} \mathrm{kpc}^{-1}$. The reader interested in a more detailed analysis of the difference among the different data sets is referred to Cols. 6 to 9 of Table 4 . 
K. Genovali et al.: On the $\alpha$-element gradients of the Galactic thin disk using Cepheids

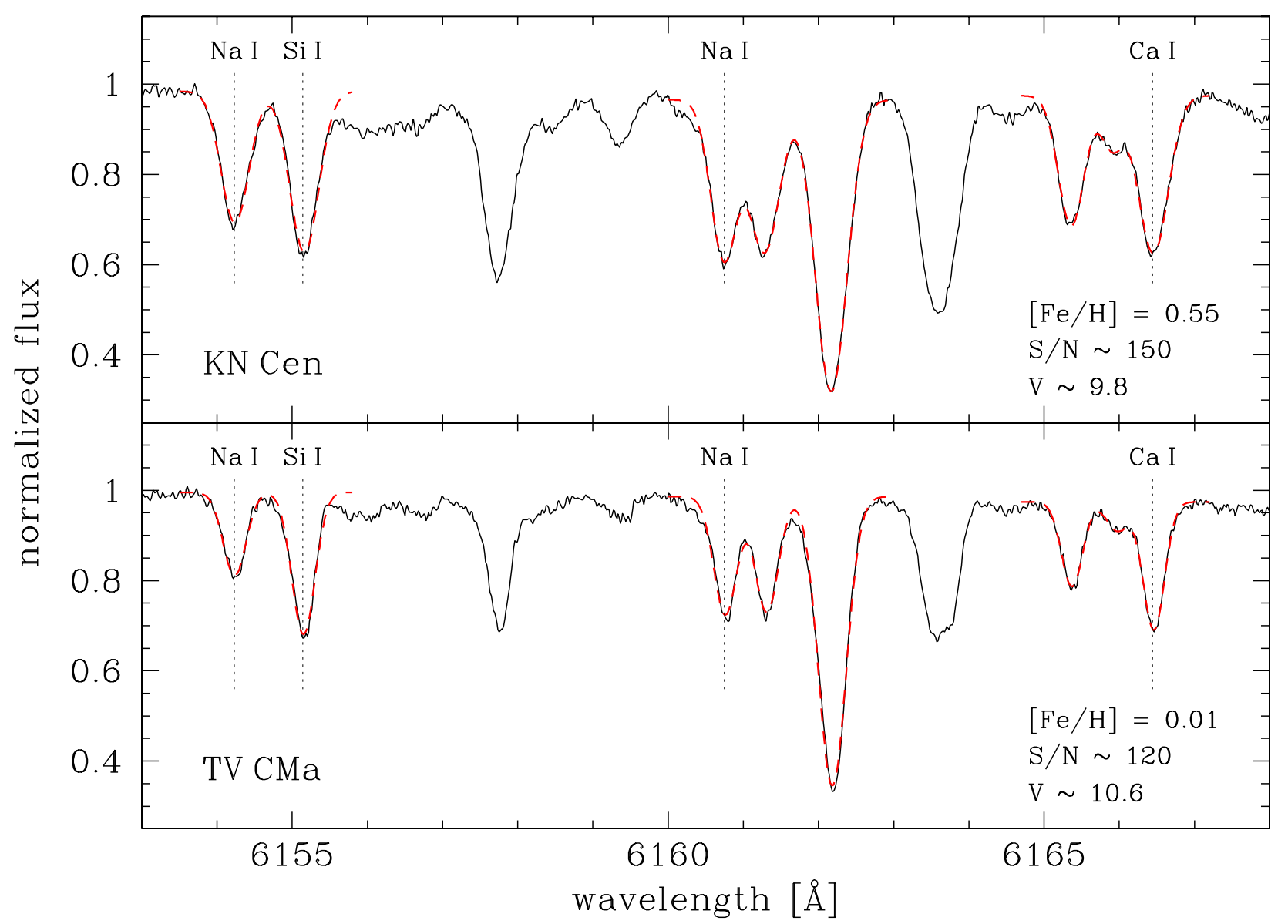

Fig. 1. High-resolution $(R \sim 38000)$ UVES spectrum of KN Cen and TV CMa. The apparent visual magnitude and the S/N in the spectral range $\lambda \sim 5650-7500 \AA$ are also labeled. The vertical dashed lines display some of the spectral lines (Na I 6154.23, Si I 6155.14, Na I 6160.75, Ca I $6166.44 \AA$ ) adopted to estimate the abundances.

Table 4. Slopes and zero-points of the abundance gradients as a function of the Galactocentric distance and of the pulsation period.

\begin{tabular}{|c|c|c|c|c|c|c|c|c|}
\hline $\begin{array}{l}\text { Abundance } \\
\text { ratio }\end{array}$ & Slope $^{a}$ & $\begin{array}{l}\text { Zero-point } \\
{[\text { dex] }}\end{array}$ & $\begin{array}{c}\sigma \\
{[\mathrm{dex}]}\end{array}$ & $N$ & Slope $^{a}(\mathrm{TS})$ & Slope $^{a}($ LEM $)$ & Slope $^{a}$ (LII) & Slope $^{a}$ (LIII) \\
\hline \multicolumn{9}{|c|}{ as a function of $R_{\mathrm{G}}$} \\
\hline$[\mathrm{Na} / \mathrm{H}]$ & $-0.052 \pm 0.003$ & $0.79 \pm 0.03$ & 0.14 & 428 & $-0.072 \pm 0.007$ & $-0.066 \pm 0.015$ & $-0.044 \pm 0.004$ & $-0.047 \pm 0.003$ \\
\hline$[\mathrm{Al} / \mathrm{H}]$ & $-0.055 \pm 0.003$ & $0.64 \pm 0.03$ & 0.13 & 426 & $-0.073 \pm 0.007$ & $-0.046 \pm 0.013$ & $-0.053 \pm 0.004$ & $-0.049 \pm 0.003$ \\
\hline$[\mathrm{Mg} / \mathrm{H}]$ & $-0.045 \pm 0.004$ & $0.56 \pm 0.03$ & 0.17 & 417 & $-0.039 \pm 0.007$ & $-0.050 \pm 0.013$ & $-0.048 \pm 0.006$ & $-0.048 \pm 0.004$ \\
\hline$[\mathrm{Si} / \mathrm{H}]$ & $-0.049 \pm 0.002$ & $0.59 \pm 0.02$ & 0.09 & 432 & $-0.055 \pm 0.005$ & $-0.068 \pm 0.009$ & $-0.049 \pm 0.003$ & $-0.048 \pm 0.002$ \\
\hline$[\mathrm{Ca} / \mathrm{H}]$ & $-0.039 \pm 0.002$ & $0.42 \pm 0.02$ & 0.11 & 434 & $-0.029 \pm 0.005$ & $-0.044 \pm 0.012$ & $-0.040 \pm 0.004$ & $-0.041 \pm 0.003$ \\
\hline$[\mathrm{Na} / \mathrm{Fe}]$ & $0.007 \pm 0.002$ & $0.23 \pm 0.02$ & 0.11 & 428 & $-0.015 \pm 0.005$ & $-0.026 \pm 0.011$ & $0.011 \pm 0.003$ & $0.015 \pm 0.002$ \\
\hline$[\mathrm{Al} / \mathrm{Fe}]$ & $0.001 \pm 0.002$ & $0.09 \pm 0.02$ & 0.10 & 425 & $-0.020 \pm 0.006$ & $0.001 \pm 0.012$ & $0.004 \pm 0.002$ & $0.012 \pm 0.002$ \\
\hline$[\mathrm{Mg} / \mathrm{Fe}]$ & $0.013 \pm 0.003$ & $0.01 \pm 0.03$ & 0.15 & 415 & $0.015 \pm 0.006$ & $0.003 \pm 0.010$ & $0.008 \pm 0.005$ & $0.010 \pm 0.003$ \\
\hline$[\mathrm{Si} / \mathrm{Fe}]$ & $0.009 \pm 0.001$ & $0.04 \pm 0.01$ & 0.06 & 432 & $0.000 \pm 0.003$ & $-0.011 \pm 0.007$ & $0.006 \pm 0.002$ & $0.012 \pm 0.001$ \\
\hline$[\mathrm{Ca} / \mathrm{Fe}]$ & $0.018 \pm 0.002$ & $-0.12 \pm 0.02$ & 0.10 & 433 & $0.028 \pm 0.004$ & $0.010 \pm 0.010$ & $0.017 \pm 0.002$ & $0.020 \pm 0.002$ \\
\hline \multicolumn{9}{|c|}{ as a function of $\log P$} \\
\hline$[\mathrm{Na} / \mathrm{H}]$ & $0.20 \pm 0.03$ & $0.16 \pm 0.02$ & 0.17 & 427 & $0.47 \pm 0.09$ & $0.23 \pm 0.08$ & $0.18 \pm 0.03$ & $0.10 \pm 0.03$ \\
\hline$[\mathrm{Al} / \mathrm{H}]$ & $0.25 \pm 0.03$ & $-0.06 \pm 0.02$ & 0.17 & 427 & $0.49 \pm 0.09$ & $0.19 \pm 0.07$ & $0.23 \pm 0.03$ & $0.13 \pm 0.03$ \\
\hline$[\mathrm{Mg} / \mathrm{H}]$ & $0.13 \pm 0.03$ & $0.04 \pm 0.03$ & 0.19 & 417 & $0.18 \pm 0.07$ & $0.24 \pm 0.09$ & $0.09 \pm 0.04$ & $0.07 \pm 0.04$ \\
\hline$[\mathrm{Si} / \mathrm{H}]$ & $0.16 \pm 0.02$ & $0.02 \pm 0.02$ & 0.14 & 435 & $0.28 \pm 0.06$ & $0.17 \pm 0.07$ & $0.14 \pm 0.03$ & $0.06 \pm 0.02$ \\
\hline$[\mathrm{Ca} / \mathrm{H}]$ & $0.09 \pm 0.02$ & $0.00 \pm 0.02$ & 0.13 & 432 & $0.16 \pm 0.05$ & $0.05 \pm 0.07$ & $0.05 \pm 0.03$ & $0.07 \pm 0.03$ \\
\hline$[\mathrm{Al} / \mathrm{Fe}]$ & $0.05 \pm 0.02$ & $0.07 \pm 0.02$ & 0.10 & 423 & $0.15 \pm 0.05$ & $0.03 \pm 0.07$ & $0.04 \pm 0.02$ & $0.06 \pm 0.02$ \\
\hline$[\mathrm{Si} / \mathrm{Fe}]$ & $-0.04 \pm 0.01$ & $0.15 \pm 0.01$ & 0.07 & 431 & $-0.04 \pm 0.03$ & $-0.02 \pm 0.04$ & $-0.05 \pm 0.01$ & $-0.04 \pm 0.01$ \\
\hline$[\mathrm{Ca} / \mathrm{Fe}]$ & $-0.11 \pm 0.02$ & $0.13 \pm 0.02$ & 0.11 & 436 & $-0.15 \pm 0.04$ & $-0.10 \pm 0.05$ & $-0.04 \pm 0.02$ & $-0.10 \pm 0.02$ \\
\hline
\end{tabular}

Notes. ${ }^{(a)}$ In units of dex kpc ${ }^{-1}$ if in function of $R_{\mathrm{G}}$, and dex per logarithmic day if in function of $\log P$. Columns from 2 to 5 shows the results for all the different samples fitted together. We also list the standard deviation $(\sigma)$ of the residuals and the number of data points $(N)$ used in the fit. The slopes using only the stars of our sample (TS: this study) and of previous studies (LEM, LII, and LIII) are shown for comparison. 


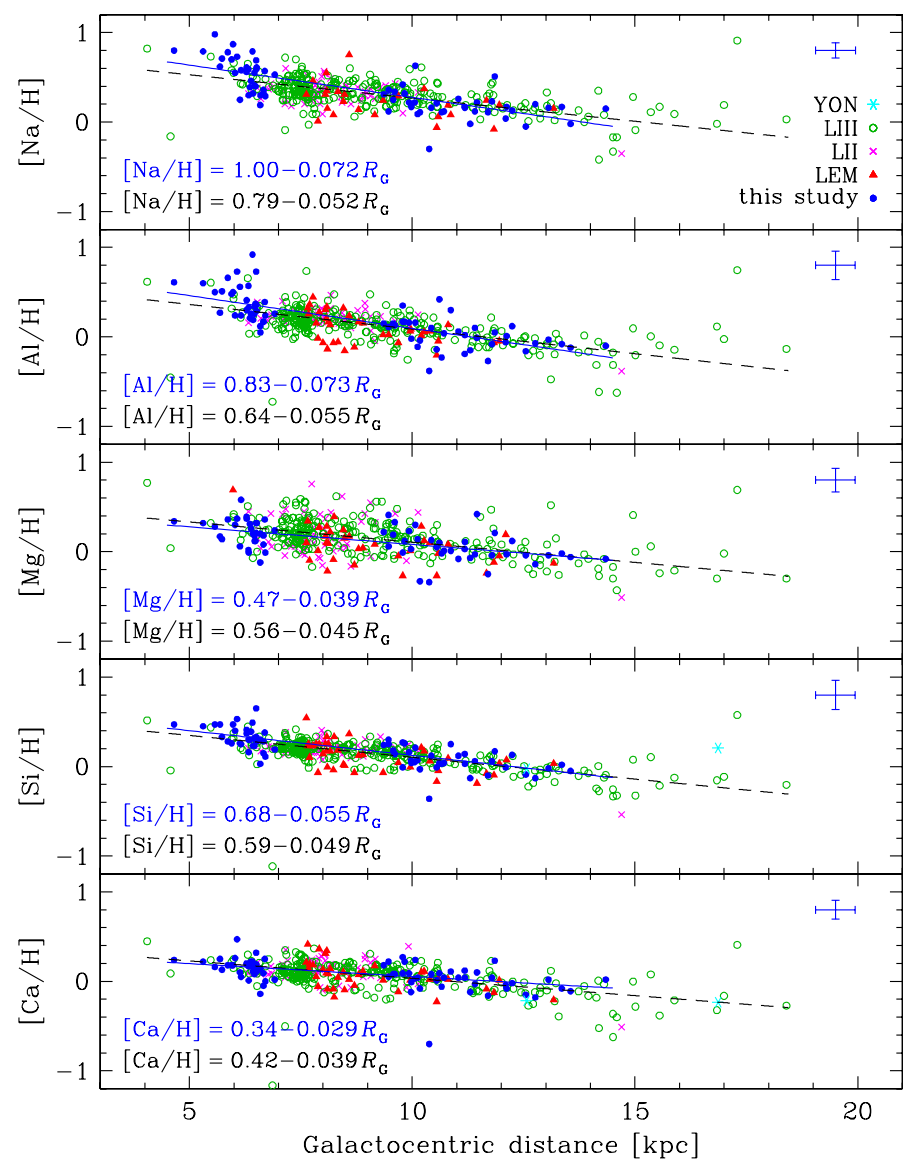

Fig. 2. Abundances of $\mathrm{Na}, \mathrm{Al}$, and $\alpha$ elements as a function of $R_{\mathrm{G}}$. Our results (filled blue circles) are compared with those of Yong et al. (2006, YON, cyan asterisks), Luck et al. (2011, LII, magenta crosses), Luck \& Lambert (2011, LIII, open green circles), and Lemasle et al. (2013, LEM, red triangles). The blue solid line shows the linear regression of our Cepheid sample, and the black dashed line the linear regression of the entire Cepheid sample. The blue error bars display the mean spectroscopic error of the current sample. The abundances available in the literature have similar errors.

\subsection{Comparisons with independent radial gradients}

Figure 3 shows the comparison between the slopes of the $\alpha$-element gradients based on Cepheids and similar abundances for Galactic field stars. We focused our attention on the $\alpha$-element abundances provided by Davies et al. (2009a,b) for two red supergiants (RSGs) located in the Galactic center and two RSGs in the Scutum cluster, plus two luminous blue variables belonging to the Quintuplet cluster measured by Najarro et al. (2009). Their $\alpha$-element and iron abundances were rescaled to the abundances of the solar mixture adopted in the current investigation (Grevesse et al. 1996). Data plotted in this figure indicate that young stellar tracers located in the innermost Galactic regions attain $\alpha$-element abundances that are, at fixed Galactocentric distance, lower than the radial gradients of Galactic Cepheids. To further constrain this difference, we extrapolated the Cepheids gradient to the Galactocentric distance of the above targets. We found that the difference $\Delta\left[\frac{1}{3}([\mathrm{Mg} / \mathrm{H}]+[\mathrm{Si} / \mathrm{H}]+[\mathrm{Ca} / \mathrm{H}])\right]$ between the expected Cepheid gradient and the Scutum stars is $0.3-0.6$ dex and becomes of the range of $0.1-0.4$ dex for the targets located in the Galactic center.

The $\alpha$-element abundances provided by these authors are based on a different approach (spectrum-synthesis vs. EWs). To

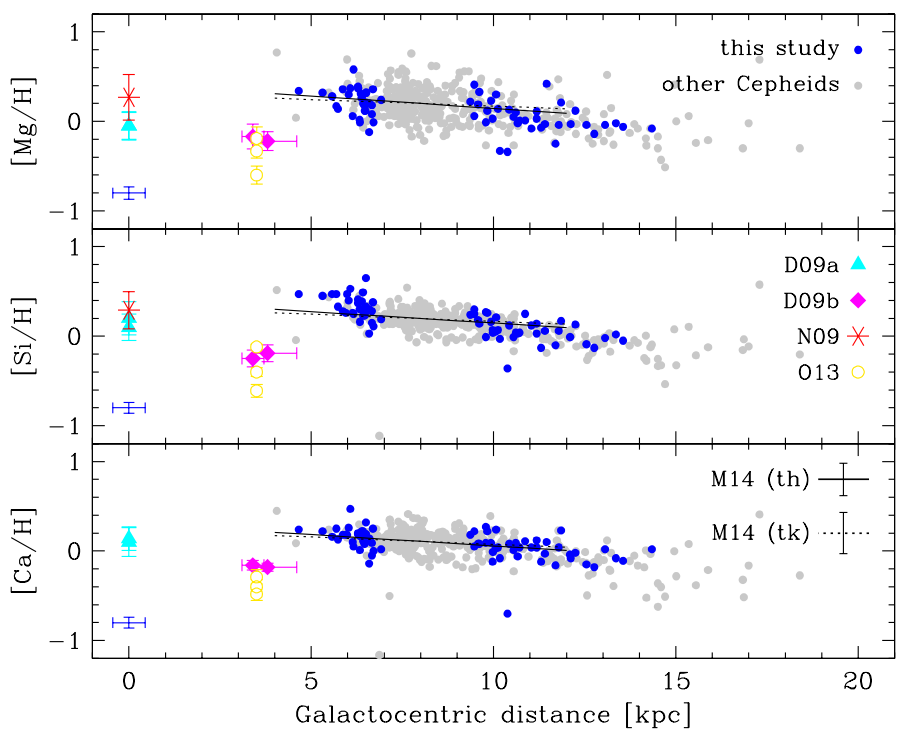

Fig. 3. Abundances of $\alpha$ elements as a function $R_{\mathrm{G}}$. Cepheid stars (filled circles) are compared with RSGs in the Galactic center analyzed by Davies et al. (2009a, D09a, cyan triangles), RSGs in the Scutum cluster analyzed by Davies et al. (2009b, D09b, magenta diamonds) and by Origlia et al. (2013, O13, yellow open circles), and with the mean abundance of two luminous blue variables (LBVs) in the Quintuplet cluster measured by Najarro et al. (2009, N09, red asterisk). The radial gradients derived by Mikolaitis et al. (2014, M14) for thin (solid line) and thick (dotted line) disk stars in the Gaia-ESO survey are also shown.

constrain the possible occurrence of systematic differences in the abundances we also plotted the $\alpha$-element abundances recently provided by Origlia et al. (2013) using high spectral resolution $(R \sim 50000)$ NIR $(Y, J, H, K)$ spectra collected with GIANO at the Telescopio Nazionale Galileo (TNG). These authors observed the same targets as observed by Davies et al. (2009b). The comparison shows, once again, that RSGs located in the near end of the Galactic bar have $\alpha$ abundances lower than the classical Cepheids located in the inner edge of the Galactic thin disk.

Figure 3 also shows the comparison with the radial gradients provided by Mikolaitis et al. (2014, hereafter M14) using thin and thick disk field stars observed within the Gaia-ESO survey. We adopted their clean sample, which only includes dwarfs, with $\log g>3.5$. To overcome possible differences between Cepheid and spectroscopic distances of field dwarfs, in plotting their radial gradients, we adopted the zero points of our gradients at the solar Galactocentric distance. The comparison shows that the slopes agree quite well over the entire range of Galactocentric distances covered by the Gaia-ESO survey (4-12 kpc).

\subsection{Age dependence of the $[\alpha / H]$ ratios}

The above findings rely on the Galactocentric distance as an independent variable. This is a crucial parameter for identifying possible radial trends. However, one of the key issues in dealing with the chemical enrichment history of the thin disk is the age dependence. Detailed chemo-dynamical models suggest a strong dependence of the metallicity gradients for ages older than 1-3 Gyr (Kubryk et al. 2015a,b; Minchev et al. 2013, 2014). This means a steady decrease in the metallicity with increasing age. Moreover, they also predict a strong dependence on stellar migrations.

Classical Cepheids can play a crucial role in this context, since there are solid theoretical and empirical arguments, 
K. Genovali et al.: On the $\alpha$-element gradients of the Galactic thin disk using Cepheids

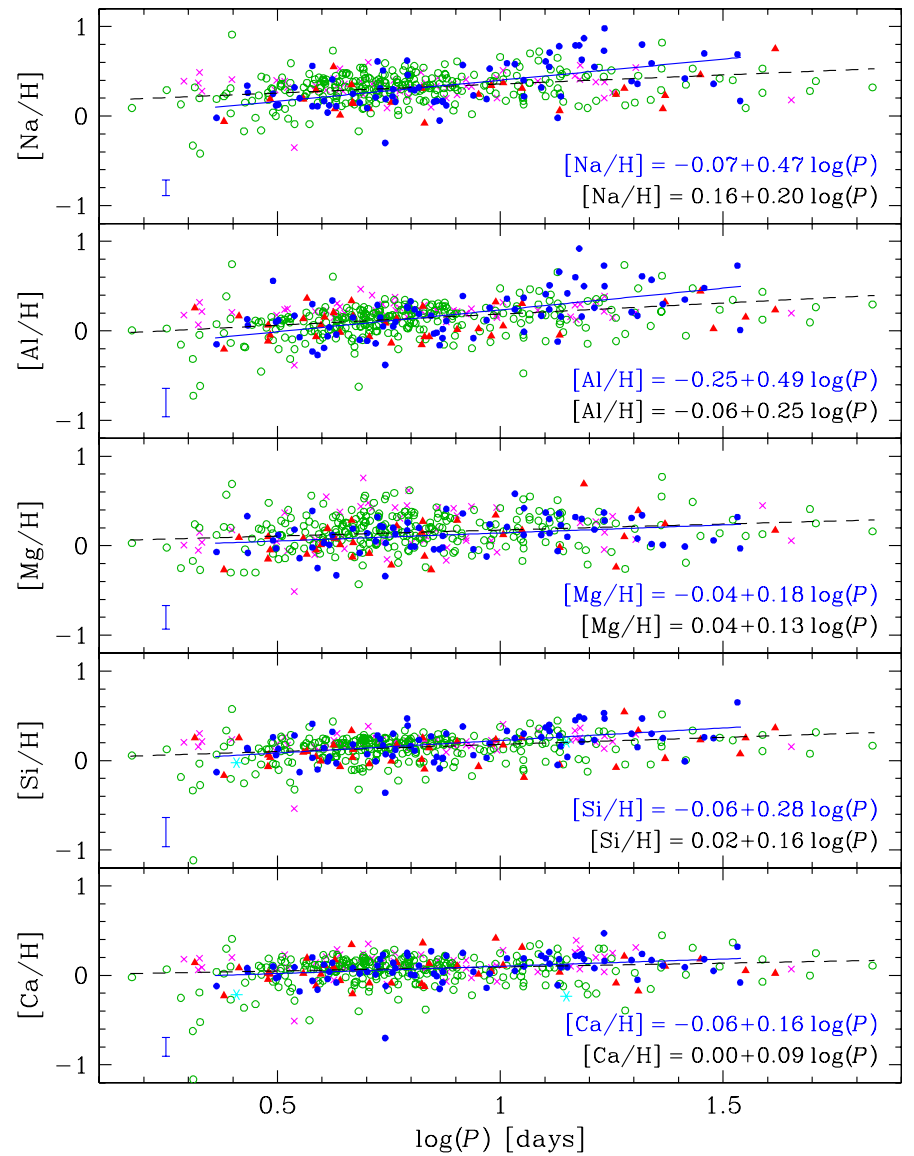

Fig. 4. Abundances of $\mathrm{Na}, \mathrm{Al}$, and $\alpha$ elements as a function of the pulsation period. Symbols and colors are the same as in Fig. 2.

suggesting that the pulsation period is strongly anticorrelated with age (Bono et al. 2005). For intermediate-mass stars during central helium-burning phases, an increase in stellar mass causes an increase in the mean luminosity of the blue loop. Plain physical arguments rooted in the Stefan-Boltzman relation, in the fundamental pulsation relation, and in the mass-luminosity relation indicate that the increase in luminosity causes a decrease in surface gravity and, in turn, an increase in the pulsation period.

To constrain the age dependence of the metallicity gradients, Fig. 4 shows the same elemental abundances as plotted in Fig. 2, but as a function of the logarithmic period. Data plotted in this figure show a well-defined positive gradient as a function of the pulsation period. The slopes of the $\alpha$-elements attain similar values, while for $\mathrm{Na}$ and $\mathrm{Al}$ they are systematically larger (see labeled values). This evidence further supports the hydrostatic nature of both $\mathrm{Na}$ and $\mathrm{Al}$ owing to their steady increase with pulsation period (stellar mass). This finding agrees quite well with yields predicted by nucleosynthesis models (Arnett 1971; Limongi \& Chieffi 2012).

\subsection{Radial gradient of [element/Fe]}

The similarity of the slopes between iron and the current elements suggested an investigation of the [element/Fe] radial gradients as a function of the Galactocentric distance. Data plotted in Fig. 5 show that the ratio is on average quite flat across the entire thin disk. We performed a biweight linear least squares fit over the entire sample, and we found that the slopes are vanishing for $\mathrm{Na}, \mathrm{Al}$, and $\mathrm{Si}$. There is mild evidence of a positive slope

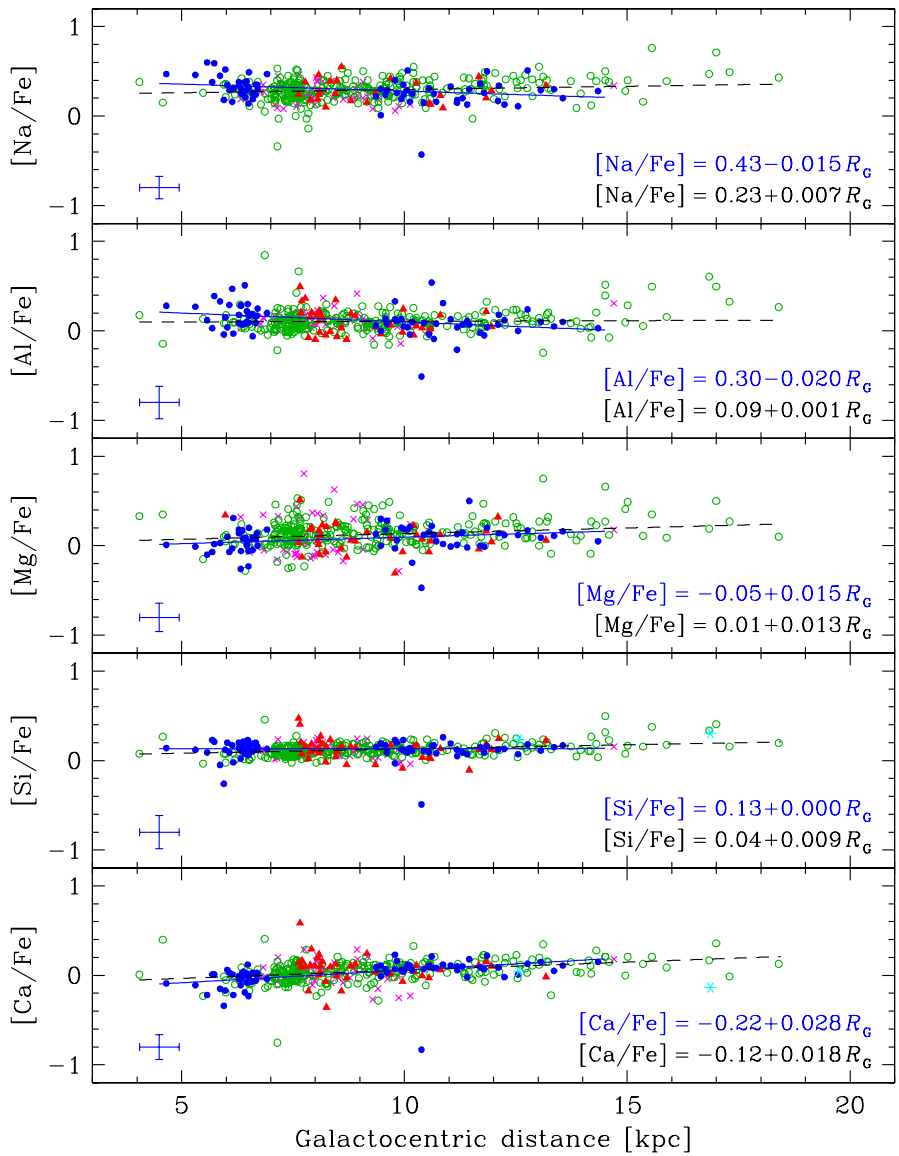

Fig. 5. Same as Fig. 2, but the abundances are scaled to iron.

for $\mathrm{Mg}$ and $\mathrm{Ca}$. However, the standard deviation of the former element is a factor of two greater than for $\mathrm{Si}(0.15 \mathrm{vs} .0 .06 \mathrm{dex})$. This suggests that the slope has to be treated cautiously. The positive slope for Ca appears more solid (see values in Table 3 ), and indeed, the slope attains the highest value among the investigated elements.

The above findings bring out a few interesting consequences:

i) The radial gradients of the [element/Fe] ratios are slightly positive if not zero. This evidence indicates that the chemical enrichment history of iron and of the other five elements has been quite similar across the Galactic thin disk. This finding is also supported by the small standard deviations of the quoted ratios. The standard deviation of $\mathrm{Si}$, the element with the most accurate measurements, can be explained as the consequence of the intrinsic error. These results become even more compelling if we take the range in iron abundance covered by Galactic Cepheids ( $\sim 1$ dex) into account.

ii) The $[\alpha / \mathrm{Fe}]$ ratios are typically considered as tracers of the star formation activity. This means that the $[\alpha / \mathrm{Fe}]$ ratios are also tracers of stellar mass and, in particular, of gas and dust mass. The lack of a clear negative gradient between the high (inner) and the low (outer) density regions of the thin disk suggests that the $[\alpha / \mathrm{Fe}]$ radial gradients are affected by other parameters, such as radial migration of stars or radial gas flows.

iii) There is evidence of a mild enhancement in the investigated elements and of an increase in the intrinsic scatter when moving toward the outer disk. The synthesis of $\mathrm{Na}$, $\mathrm{Al}$, and $\mathrm{Mg}$ takes place mainly in massive stars during hydrostatic central He-, C-, or Ne-burning phases and current 


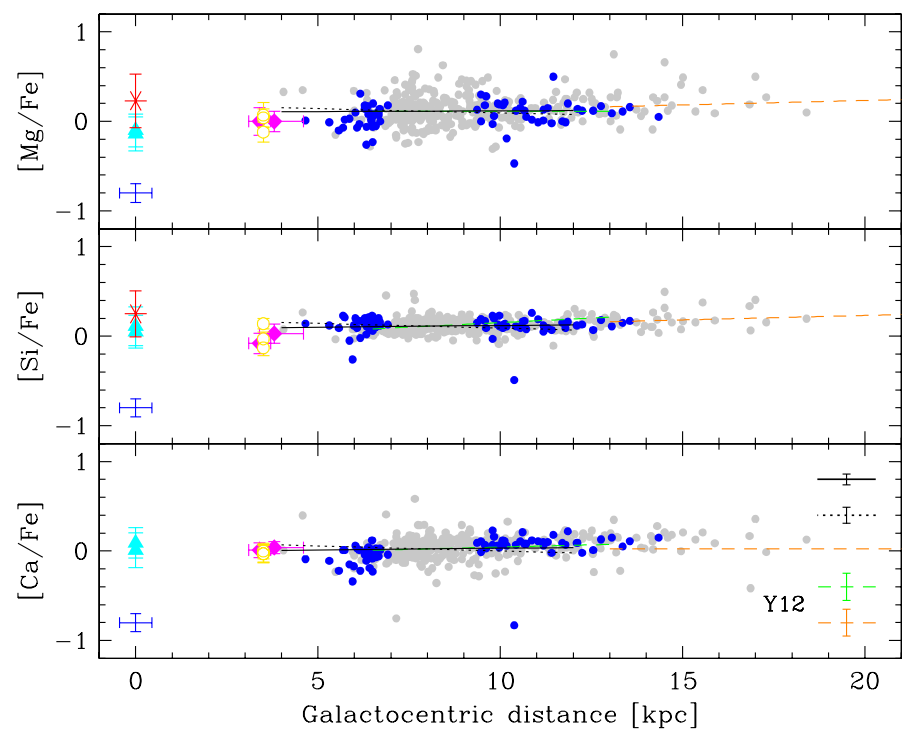

Fig. 6. Same as Fig. 3, but the abundances are scaled to iron. The abundance trends derived by Yong et al. (2012, Y12) for open clusters (green and orange dashed lines) are also shown.

predictions suggest that their production depends on metallicity (Woosley \& Weaver 1995; Limongi \& Chieffi 2012). However, these findings further support the evidence that the ratios $[\mathrm{Na} / \mathrm{Fe}]$ and $[\mathrm{Al} / \mathrm{Fe}]$ are constant across the Galactic thin disk (McWilliam et al. 2013).

However, the number of Cepheids with Galactocentric distance larger than $13 \mathrm{kpc}$ is limited. New identifications of classical Cepheids in the outer disk from the ongoing long-term photometric surveys are clearly required to further constrain the trend in the outer disk. The same applies for new homogenous spectroscopic measurements.

\subsection{Comparisons with independent radial gradients}

To validate the above findings concerning the flat trend of $[\alpha / \mathrm{Fe}]$ radial gradients, Fig. 6 shows the comparison with similar data available in the literature. The colored symbols denote the same young stars plotted in Fig. 3. Interestingly enough, $[\alpha / \mathrm{Fe}]$ is solar and quite similar to the mean ratio of the entire Cepheid sample. This evidence further supports the working hypothesis that the Galactic thin disk experienced a homogeneous chemical enrichment history during the last $300 \mathrm{Myr}$. The lack of a clear enhancement in the innermost Galactic regions is also supporting the mild correlation of the $[\alpha / \mathrm{Fe}]$ ratio with the star formation rate. Indeed, recent NIR (Matsunaga et al. 2011) and MIR/FIR (Ramírez et al. 2008; Gerin et al. 2015) investigations indicate that the Galactic bar and the Galactic center are very efficient star-forming regions.

A comparison with radial gradients provided by M14, for thin and thick disk field stars in the Gaia-ESO survey further supports the above conclusions. Indeed, there is very good agreement with the $[\alpha / \mathrm{Fe}]$ ratios of both thin and thick disk stars. Once again, in plotting their radial gradients we adopted the zero points of our gradients at the solar Galactocentric distance.

The above figure also shows the comparison with the $\alpha$-element abundances for open clusters provided by Yong et al. (2012). It shows the gradients for inner $\left(R_{\mathrm{G}}<13 \mathrm{kpc}\right)$ and outer $\left(R_{\mathrm{G}}>13 \mathrm{kpc}\right)$ disk objects. In plotting these gradients we adopted the zero points of our gradients at the solar
Galactocentric distance. The agreement is quite good over the entire range of distances. This finding becomes even more compelling if we account for the fact that the open clusters adopted by Yong et al. cover the entire range of ages typical of thin disk stellar populations, namely from $\sim 0.5$ to $\sim 10 \mathrm{Gyr}$ (see their Figs. 25 and 26).

\subsection{Age dependence of the $[\alpha / \mathrm{Fe}]$ ratios}

To constrain the age dependence of the $[\alpha / \mathrm{Fe}]$ abundance ratios, Fig. 7 shows the same elemental abundances as are plotted in Fig. 5, but as a function of the logarithmic period. A glance at the data plotted in this figure shows that the ratios are approximately constant over the entire period range. The difference in age between short- $(\log P \sim 0.3)$ and long-period $(\log P \sim 1.8)$ is on the order of $300 \mathrm{Myr}$ (Bono et al. 2000; Anderson et al. 2014). These findings strongly suggest that the chemical enrichment of Galactic Cepheids has been homogenous both in space and in time.

To constrain the period/age dependence on a more quantitative basis, we performed biweight linear least squares fits to the above data. The zero points, the slopes, their uncertainties, and the standard deviations are listed in the bottom lines of Table 4. We found that $\mathrm{Na}, \mathrm{Al}, \mathrm{Mg}$, and $\mathrm{Si}$ either do not show evidence of a slope or the slope is marginal (see Fig. 7). On the other hand, $\mathrm{Ca}$ shows once again a (negative) gradient, suggesting that $\mathrm{Ca}$ is underabundant among the youngest Cepheids. In passing we note that the nucleosynthesis of $\mathrm{Si}, \mathrm{Ca}$, and $\mathrm{Fe}$ mostly takes place during the $\mathrm{SNe}$ II explosive events. These elements, in particular $\mathrm{Fe}$, are also produced in SNe Ia. This might explain the flat distribution of $\mathrm{Si}$, but the negative trend shown by $\mathrm{Ca}$ when moving from older to younger Cepheids would remain unclear. The occurrence of a negative gradient in $\mathrm{Ca}$ as a function of period and of a positive gradient of $\mathrm{Ca}$ as a function of Galactocentric distance are not correlated, since the Cepheids located in the outer disk have a canonical period distribution (mostly between 2 and 20 days).

\section{Metallicity distribution}

The range in age covered by Galactic Cepheids is too short to constrain a possible age dependence on the timescale of a few Gyrs. To further investigate this effect we compared the current Cepheid metallicity distribution with the metallicity distribution of field dwarf stars (743) collected by Soubiran \& Girard (2005, hereafter SG05). In their sample, $72 \%$ are thin disk stars and $28 \%$ are from the thick disk. The sample collected by SG05 is based on spectroscopic data available in the literature. Their $\alpha$-element and iron abundances were not rescaled to the solar mixture adopted in the current investigation (Grevesse et al. 1996). We also perform the same comparison with the results provided by the Gaia-ESO survey (Mikolaitis et al. 2014; RecioBlanco et al. 2014). Data plotted in Fig. 8 shows that the agreement between Cepheids and thin disk dwarfs is quite good over the entire metallicity range covered by the current sample. This outcome applies to the $\alpha$-elements and to Al. On the other hand, the $\mathrm{Na}$ in field stars attain values that are the lower envelope of the typical $\mathrm{Na}$ abundances of classical Cepheids. The same agreement for $\alpha$-elements and for $\mathrm{Al}$ is also shared in the [element/Fe] plane (Fig. 9), in which the difference concerning $\mathrm{Na}$ is even clearer. This difference appears to be significant and may be caused by non-LTE effects. Indeed, the difference increases when moving from the metal-rich to the metal-poor regime, as 
K. Genovali et al.: On the $\alpha$-element gradients of the Galactic thin disk using Cepheids

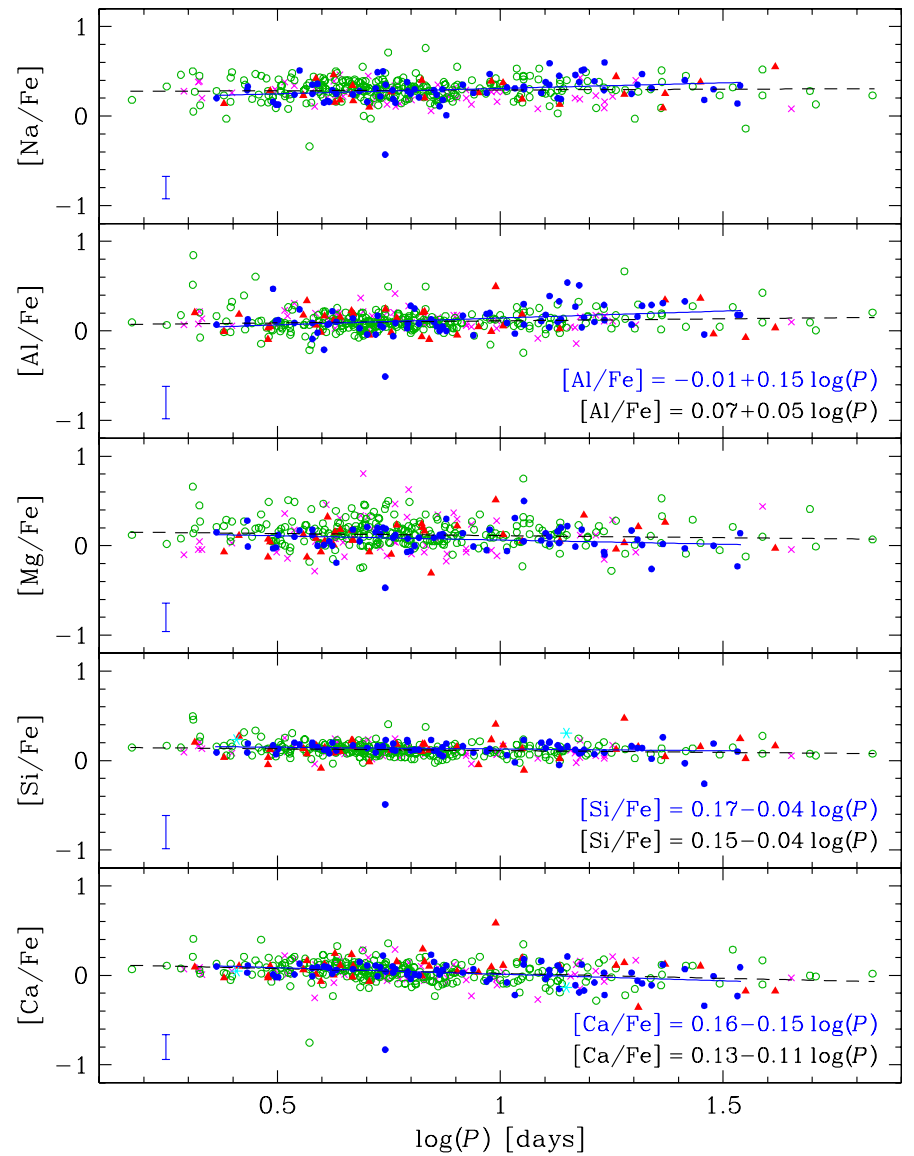

Fig. 7. Abundances of $\mathrm{Na}, \mathrm{Al}$, and $\alpha$ elements scaled to iron as a function of the logarithmic pulsation period. For $\mathrm{Na}$ and $\mathrm{Mg}$ the equations are not shown because the slopes have no statistical significance. Symbols and colors are the same as in Fig. 2.

expected for non-LTE effects (Fabrizio et al. 2012; Takeda \& Takada-Hidai 2000). Recent empirical investigations (Johnson \& Pilachowski 2012) are also suggesting an increase in $\mathrm{Na}$ enhancement when moving from the base to the tip of the RGB. Moreover, we also found evidence of a positive gradient (slope $=$ $\left.0.20 \pm 0.03 \mathrm{dex} \mathrm{kpc}^{-1}\right)$ in $[\mathrm{Na} / \mathrm{H}]$ versus the logarithmic period. This finding further supports an increase in the difference in $\mathrm{Na}$ abundance when moving from long periods (low surface gravity) to short periods (high surface gravity).

In this context it is worth mentioning that Takeda et al. (2013) performed a detailed abundance analysis of ten Galactic Cepheids using high-resolution spectra. They investigated CNO plus $\mathrm{Na}$ elements, since they are solid tracers of induced mixing during advanced evolutionary phases of intermediate-mass stars. They found a well-defined Na overabundance on the order of 0.2 dex over the entire period range covered by their targets (2-16 days). They suggested that the $\mathrm{Na}$ enhancement could be due to mixing events that dredge up Na-rich material, produced by the NeNa cycle, into the surface of classical Cepheids. A similar explanation was originally suggested by Sasselov (1986) and by Denissenkov (1994). We plan to address this specific issue and, in particular, the period (i.e., stellar mass) dependence in a forthcoming paper.

\subsection{Al-Mg and $\mathrm{Na}-\mathrm{O}$ correlations}

To further constrain the recent chemical enrichment of the Galactic thin disk, we also investigated the (anti-)correlation

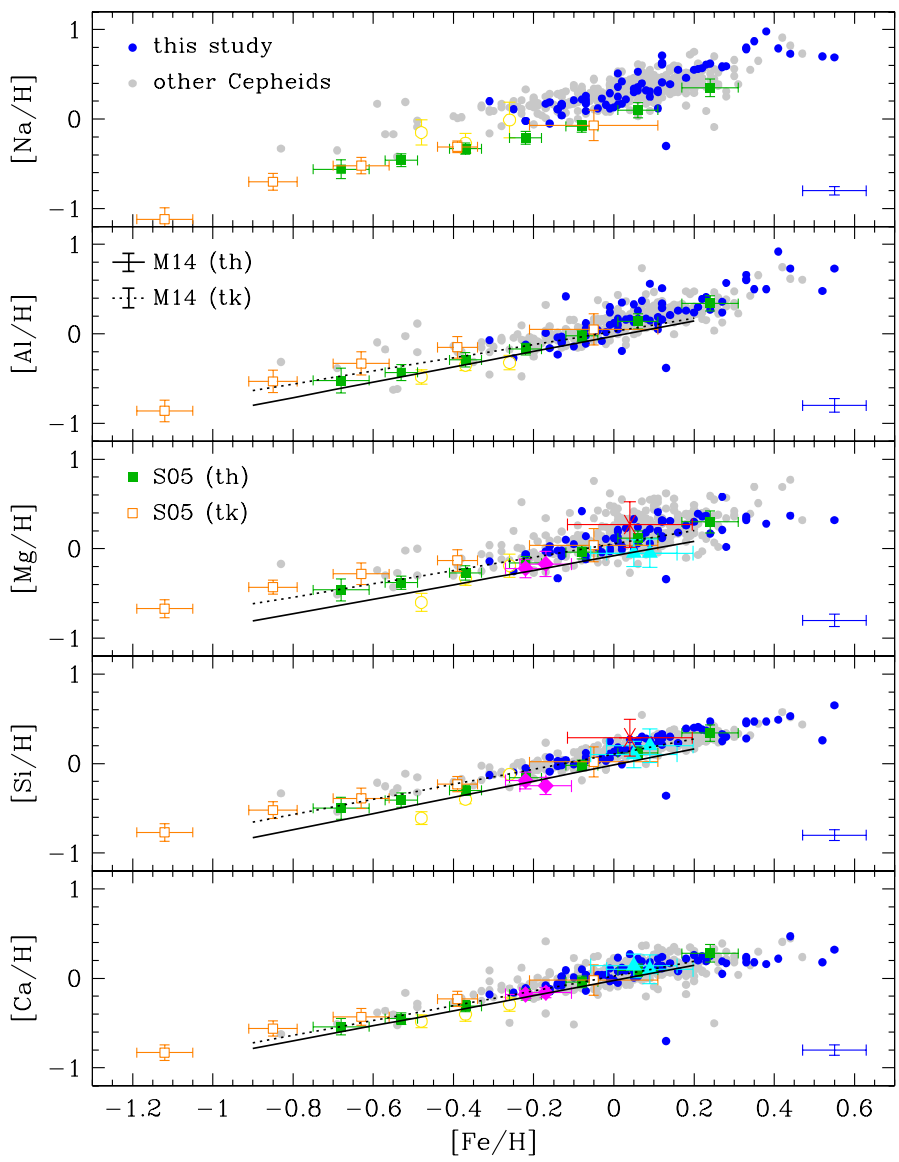

Fig. 8. Abundances of $\mathrm{Na}, \mathrm{Al}$, and $\alpha$ elements as a function of the metallicity. Cepheid stars (filled circles) are compared with thin disk (filled green squares) and thick disk (open orange squares) field dwarfs from Soubiran \& Girard (2005, S05). The abundances of red supergiants and LBVs are also plotted when available (symbols and colors are the same as in Fig. 3). The gradients derived by Mikolaitis et al. (2014, M14) for thin (solid line) and thick (dotted line) disk stars in the Gaia-ESO survey are also shown.

between $\mathrm{Mg}-\mathrm{Al}$ and $\mathrm{Na}-\mathrm{O}$. Detailed spectroscopic investigations indicate that evolved and unevolved stars in the Local Group globular clusters display well-defined anti-correlation between $\mathrm{Na}-\mathrm{O}$ and $\mathrm{Mg}-\mathrm{Al}$ (e.g., Carretta et al. 2009, 2014). The lefthand panel of Fig. 10 shows the comparison between the current abundances of $\mathrm{Mg}$ and $\mathrm{Al}$ for the Cepheid sample (blue dots plus gray dots for Cepheids in the literature) with similar abundances for F- and G-type field dwarf stars collected by Bensby et al. (2005, hereafter B05, 102 objects). The latter sample includes both thin (dark green) and thick (light green) disk stars. Moreover, we also included $\mathrm{Mg}-\mathrm{Al}$ abundances for field dwarf stars collected by Reddy et al. (2003, hereafter R03). This sample includes 189 objects and a significant fraction of them are thin disk stars (magenta dots). The $\alpha$-element and iron abundances provided by these authors were rescaled to the abundances of the solar mixture adopted in the current investigation (Grevesse et al. 1996).

Data plotted in this figure disclose the very good agreement between field dwarfs and Cepheids. However, the sample by R03 shows, at fixed $[\mathrm{Mg} / \mathrm{Fe}]$ abundance, a well-defined underabundance in $[\mathrm{Al} / \mathrm{Fe}]$. There are some plausible reasons that could explain the difference. The $\mathrm{Mg}$ abundances provided by R03 are based on three lines $(4730.04,6318.71,7657.61 \AA)$, while we only use the line at $5711.09 \AA$. The agreement with 


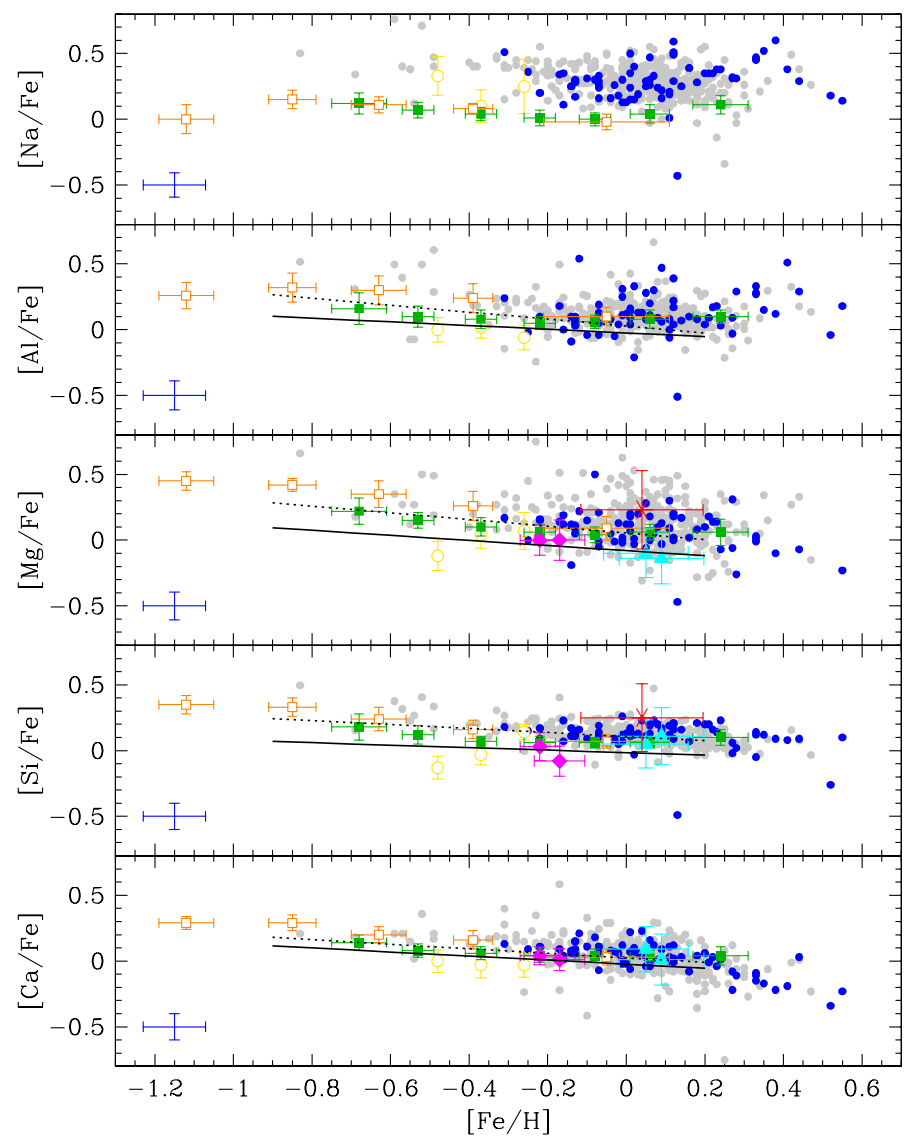

Fig. 9. Same as Fig. 8, but the abundances are scaled to iron.

the $\mathrm{Mg}$ abundances provided by B05 is because they adopted eight different lines including the current one, but not the three $\mathrm{Mg}$ lines adopted by R03. The difference may also be caused by different values of $\log g f$ adopted by these studies. Nevertheless, Galactic Cepheids display, at fixed $[\mathrm{Al} / \mathrm{Fe}]$ abundance, a large dispersion in $[\mathrm{Mg} / \mathrm{Fe}]$ abundances when compared with field dwarfs. However, they clearly follow the $\mathrm{Mg}-\mathrm{Al}$ correlation typical of field stars.

To confirm the above similarity, the middle panel of Fig. 10 shows the same comparison, but with the thin- and thick-disk field dwarf stars measured by M14. The agreement is once again very good over the entire abundance range covered by the two samples. Data plotted in this panel display that Cepheids and thin- and thick-disk dwarf stars have similar $[\mathrm{Al} / \mathrm{Fe}]$ and $[\mathrm{Mg} / \mathrm{Fe}]$ distributions. This is an interesting finding, since according to R03, B05, and M14, their samples include both old ( 12 Gyr) and intermediate-age (0.5-7 Gyr) field dwarf stars.

The righthand panel of Figure 10 shows the comparison between the abundances of $\mathrm{Na}$ and $\mathrm{O}$ for the current sample of Cepheids with the same sample of field stars as we adopted in the lefthand panel of the same figure. In plotting Cepheid abundances, we are using the current $\mathrm{Na}$ abundances, but the $\mathrm{O}$ abundances for the same objects as provided by LII and LIII. To overcome problems introduced by non-LTE effects, we only used oxygen abundances based on the O I triplet at $6156 \AA$ and on the [OI] forbidden line at $6300 \AA$, with the mean values of both provided by LIII, and the triplet-based ones provided by LII. A glance at the data plotted in this panel shows the well-defined offset in $\mathrm{Na}$ abundances between field dwarfs and Cepheids. The sample by R03 appears to be located between the B05 and the Cepheids. This mild difference might be due to their larger uncertainties in $\mathrm{O}$ abundances (see their Sect. 7.2) or to different assumptions on the adopted $\log g f$ values.

\subsection{Hydrostatic and explosive elements}

The chemical enrichment history of the Galactic thin disk can also be constrained by investigating the ratio between hydrostatic and explosive elements. The lefthand panel of Fig. 11 shows $[\mathrm{Mg} / \mathrm{Ca}]$ as a function of the iron abundance. The Cepheids display typical solar abundances of $[\mathrm{Mg} / \mathrm{Ca}]$ for iron abundances that are more metal-poor than the Sun. For iron abundances more metal-rich than the Sun, the spread in $[\mathrm{Mg} / \mathrm{Ca}]$ abundances increases and becomes on the order of 0.5-0.7 dex. On the other hand, the abundances of thin- and thick-disk dwarf stars collected either by B05 or by M14 display the typical decreasing trend when moving from metalpoor to more metal-rich objects ${ }^{4}$. The same outcome applies to the metal-rich regime, and indeed the metal-rich objects (green diamonds) collected by B05 display a mild increase in the $[\mathrm{Mg} / \mathrm{Ca}]$ abundance ratio for $[\mathrm{Fe} / \mathrm{H}] \gtrsim 0.15$.

The reasons for the above difference are not clear, therefore we investigated a possible radial dependence. The middle panel of Fig. 11 shows both the Cepheid and the M14 [Mg/Ca] abundances as a function of Galactocentric distance. Data plotted in this figure display that a significant fraction of the spread in $[\mathrm{Mg} / \mathrm{Ca}]$ abundance $(\sim 0.7 \mathrm{dex})$ showed by metal-rich Cepheids is evident among objects that are located either across or inside the solar circle $(\sim 8 \mathrm{kpc})$. Evidence of a mild increase in the spread in $[\mathrm{Mg} / \mathrm{Ca}]$ seems to also be present in the outer disk.

The thin-disk stars by M14 display a flat distribution over the entire range of the Galactocentric distances they cover. On the other hand, the thick-disk stars display a broader spread $(\sim 0.3$ dex $)$ in a limited range of Galactocentric distances $(6 \lesssim$ $R_{\mathrm{G}} \lesssim 8 \mathrm{kpc}$ ). In this panel we did not plot the sample by B05, since individual distances are not available. This evidence appears slightly counter intuitive, since the increase in iron typical of the innermost disk regions should also be followed by a steady increase in $\mathrm{Ca}$ abundance, since they are explosive elements. This means a steady decrease in the $[\mathrm{Mg} / \mathrm{Ca}]$ abundance ratio. Oddly enough, we found that the $[\mathrm{Mg} / \mathrm{Ca}]$ ratio increases when moving toward the inner disk.

To further constrain this effect, we also investigated the age dependence. The righthand panel of Fig. 11 shows the $[\mathrm{Mg} / \mathrm{Ca}]$ abundances of the Cepheid sample as a function of the logarithmic period. Data plotted in this figure show no clear trend. The spread in the $[\mathrm{Mg} / \mathrm{Ca}]$ abundance ratio is almost constant over the entire period range, so there is no solid evidence of a dependence on stellar mass.

\section{Summary and final remarks}

We have presented accurate and homogeneous measurements of $\mathrm{Na}, \mathrm{Al}$, and three $\alpha$-elements $(\mathrm{Mg}, \mathrm{Si}, \mathrm{Ca})$ for 75 classical Galactic Cepheids. The current abundances are based on high spectral resolution $(R \sim 38000)$ and a high signal-to-noise ratio $(S / N \sim 50-300)$ spectra collected with UVES at ESO VLT. The iron abundance of the same Cepheids was already discussed in Genovali et al. $(2013,2014)$. The current sample covers a broad range in pulsation periods $(0.36 \leq \log P \lesssim 1.54)$ and in Galactocentric distances $\left(4.6 \leq R_{\mathrm{G}} \leq 14.3 \mathrm{kpc}\right)$.

\footnotetext{
4 The thin-disk stars by M14 appear to have a flat distribution, but we performed a test and found that the negative slope is significant.
} 
K. Genovali et al.: On the $\alpha$-element gradients of the Galactic thin disk using Cepheids
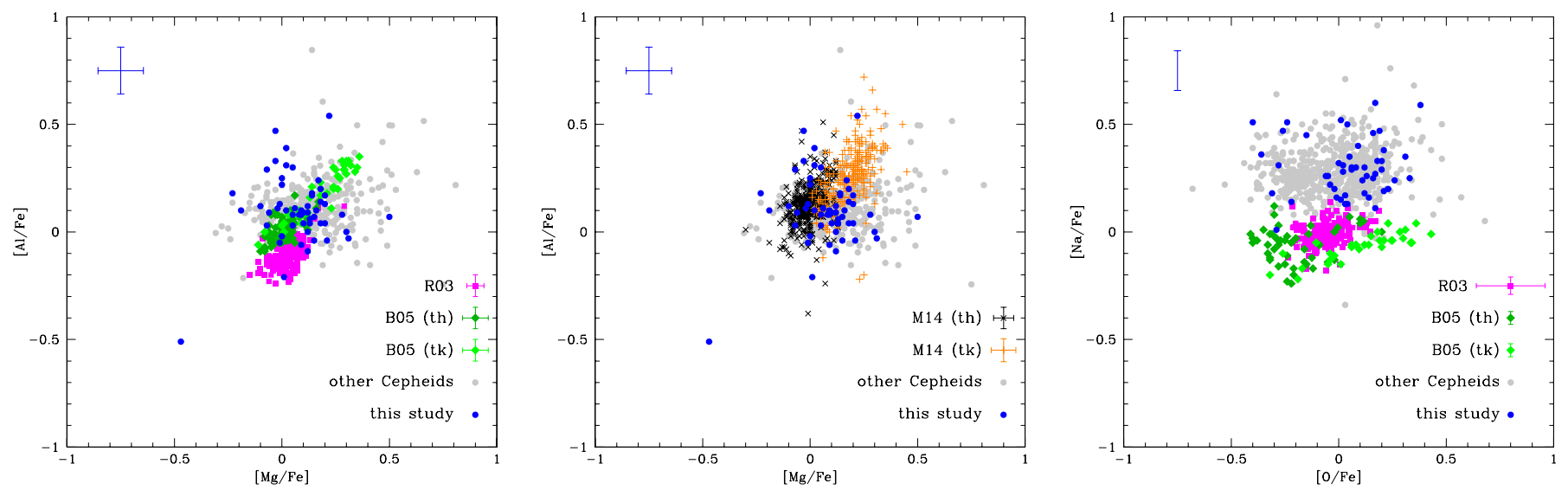

Fig. 10. Correlations between $\mathrm{Al}-\mathrm{Mg}$ (left and middle panels) and between $\mathrm{Na}-\mathrm{O}$ (right panel). Cepheid stars (filled circles) are compared with field dwarfs from the thin disk analyzed by Reddy et al. (2003, R03, magenta squares), from the thin (dark green diamonds) and thick (light green diamonds) disks analyzed by Bensby et al. (2005, B05), and from the thin (black crosses) and thick (orange pluses) disks analyzed by Mikolaitis et al. (2014, M14).
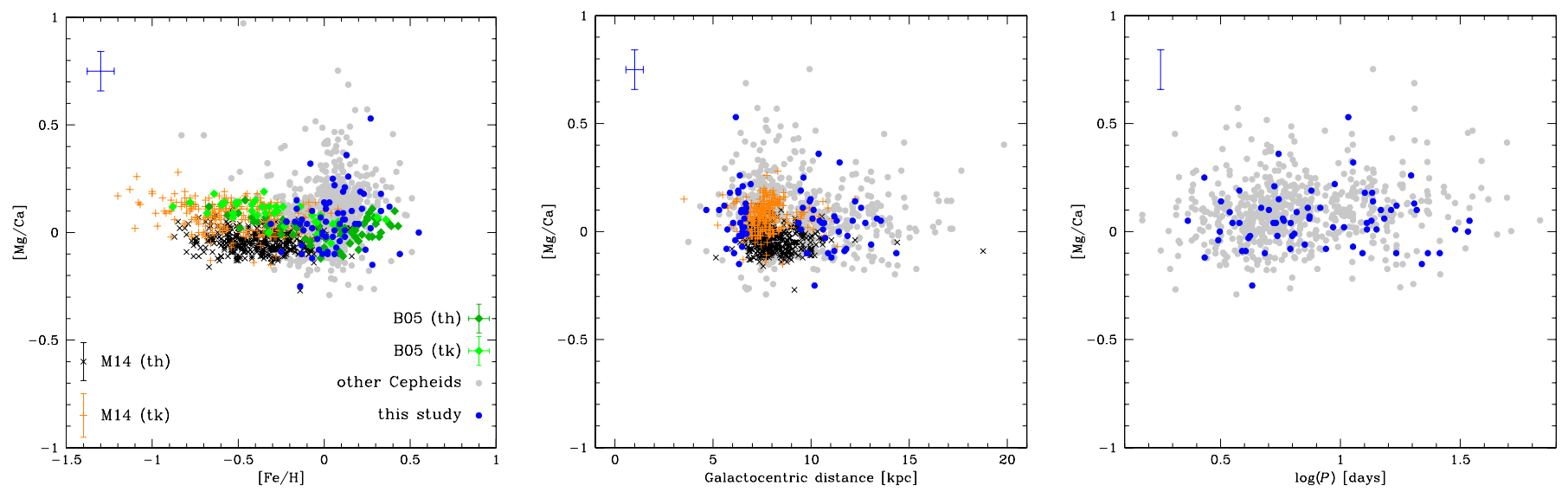

Fig. 11. Abundance ratios between $\mathrm{Mg}$ and $\mathrm{Ca}$ as a function of metallicity (left panel), Galactocentric distance (middle panel), and logarithmic pulsation period (right panel). Symbols and colors are the same as in Fig. 10.

The current spectroscopic measurements were complemented with Cepheid abundances available in the literature and based on similar spectra and on a similar approach in performing the abundance analysis. We ended up with a sample of 439 Galactic Cepheids. Among them 140 have measurements estimated by our group (current plus LEM), while the others come from LII, LIII, and YON. The samples have from 16 to 55 Cepheids in common, which allow us to calibrate a homogeneous abundance scale for the quoted five elements plus iron $\mathrm{s}(\mathrm{G} 14)$.

The use of homogeneous abundance scales and accurate and homogenous individual distances based on NIR photometry allowed us to investigate the radial gradients across a significant portion of the Galactic inner and outer disks $\left(4.1 \leq R_{\mathrm{G}} \leq\right.$ $18.4 \mathrm{kpc}$ ). The main findings of the current analysis are the following:

i) Radial gradients: the five investigated elements display a well-defined radial gradient. The slopes range from -0.058 for $[\mathrm{Al} / \mathrm{H}]$ to $-0.038 \mathrm{dex} \mathrm{kpc}^{-1}$ for $[\mathrm{Ca} / \mathrm{H}]$. The negative slopes are linear over the entire range of Galactocentric distances covered by the current sample. Moreover, they agree, within the errors, with similar estimates available in the literature. The main difference is that the current gradients display standard deviations that are on average smaller than 0.16 dex.

ii) Environmental effects: the comparison of current abundances with similar measurements for young stars located at the near end of the bar and in the nuclear bulge indicates that the latter attain solar abundances, within the errors. This means that inner-disk Cepheids attain higher $\alpha$ abundances than do young stars of the quoted regions. Thus suggests that the bar, the nuclear bulge, and the inner disk underwent different chemical enrichment histories. However, the above regions attain similar solar $[\alpha / \mathrm{Fe}]$ ratios.

iii) Spatial homogeneity: the [element/Fe] ratios are constant across the entire thin disk with no evidence of a radial gradient. This applies to $\mathrm{Na}, \mathrm{Al}$, and $\mathrm{Si}$. The radial distribution of $\mathrm{Mg}$ and $\mathrm{Ca}$ display marginal positive slopes, but they should be treated with caution. The Mg abundances display, at fixed Galactocentric distance, a wide spread mainly driven by measurement errors.

The above results indicate that the chemical enrichment history of iron and of the five other elements has been quite similar across the Galactic thin disk. Moreover, since the $[\alpha / \mathrm{Fe}]$ ratios are typically considered tracers of star formation, the lack of a clear negative gradient suggests that the $[\alpha / \mathrm{Fe}]$ radial gradients are also affected by other parameters. 
There is also evidence of a mild abundance enhancement for the five elements and of an increase in the intrinsic scatter in the outer disk. However, the number of Cepheids with $R_{\mathrm{G}}>13 \mathrm{kpc}$ is limited, and new identifications of classical Cepheids in this region are clearly required.

iv) Temporal homogeneity: the ratios [element/Fe] vs. $\log P$ are constant over the entire period range for $\mathrm{Na}, \mathrm{Al}$, and $\mathrm{Mg}$. This empirical evidence, together with the well-established anti-correlation between age and pulsation period, indicates that the chemical enrichment of Galactic Cepheids has also been very homogenous during the past $300 \mathrm{Myr}$. On the other hand, Ca shows a significant negative gradient, since it is underabundant among the youngest Cepheids.

v) Comparison between giants and dwarfs: the comparison between the metallicity distribution of Galactic Cepheids and field dwarf stars shows that the current $\mathrm{Al}, \mathrm{Mg}, \mathrm{Si}$, and $\mathrm{Ca}$ abundances agree quite well with those of the thin disk dwarfs over the entire metallicity range. On the other hand, the $\mathrm{Na}$ abundances in field stars are the lower envelope of the typical $\mathrm{Na}$ abundances of classical Cepheids. This effect appears real and caused by non-LTE effects, since the difference increases when moving from the metal-rich to metalpoor Cepheids. More recent investigations suggest that an increase in $\mathrm{Na}$ abundance in evolved intermediate-mass stars might also be caused by mixing events that dredge Na-rich material up, which is produced by the $\mathrm{NeNa}$ cycle, into the surface level of classical Cepheids.

vi) $\mathrm{Mg}-\mathrm{Al}$ and $\mathrm{Na}-\mathrm{O}$ correlations: evolved and unevolved stars in the Local Group globular clusters show a well-defined anti-correlation between $\mathrm{Mg}-\mathrm{Al}$ and $\mathrm{Na}-\mathrm{O}$. On the other hand, classical Cepheids follow the same correlations as are typical of field stars. However, Cepheid $\mathrm{Na}$ abundances show a well-defined enhancement when compared with $\mathrm{Na}$ abundances for field dwarfs. The difference is mainly caused by evolutionary effects (dredge-up into the surface of Na-rich material) and by non-LTE effects. Moreover, Cepheids, thinand thick-disk dwarf stars display very similar $[\mathrm{Al} / \mathrm{Fe}]$ and $[\mathrm{Mg} / \mathrm{Fe}]$ abundance distributions. This is an interesting finding since the different samples of field dwarfs and Cepheids cover a wide range in age $(0.5-12 \mathrm{Gyr})$.

vii) Hydrostatic and explosive elements: Cepheids in the $[\mathrm{Mg} / \mathrm{Ca}]$ vs. $[\mathrm{Fe} / \mathrm{H}]$ plane display a flat solar abundance for iron abundances that are more metal-poor than the Sun. For iron abundances more metal-rich than the Sun, the spread in $[\mathrm{Mg} / \mathrm{Ca}]$ abundances increases and reaches about 0.5-0.7 dex. Thin- and thick-disk stars display similar trends in the quoted iron regimes. The $[\mathrm{Mg} / \mathrm{Ca}]$ abundance ratio decreases, approaching solar iron abundances, and increases in the more metal-rich regime. Simple chemical enrichment histories would imply a steady decrease in $[\mathrm{Mg} / \mathrm{Ca}]$ for higher $\mathrm{Fe}$ abundances, since $\mathrm{Ca}$ and $\mathrm{Fe}$ are explosive elements. This trend is mainly caused by objects located either across from or inside the solar circle and does not show a clear dependence on the pulsation period (i.e., stellar mass).

The above results bring out two interesting consequences concerning the chemical enrichment of the Galactic thin disk: the flat and homogeneous trend of the five investigated elements as a function of the Galactocentric distances and of the pulsation period. This suggests similar trends when moving from the inner to the outer disk. Moreover, there is mounting evidence of a marginal difference between young (Cepheids), intermediateage, and old field dwarfs. This means minor spatial and temporal variations across the Galactic thin, and possibly thick, disk.
More solid empirical constraints are required to further support the above trends. There is no doubt that $s$ - and $r$-elements are good diagnostics for constraining possible differences in chemical enrichment between different stellar populations (Cescutti et al. 2008; Matteucci et al. 2014).

The wealth of new results concerning iron and $\alpha$-elements is also opening the path to new empirical constraints on the initial mass function. In particular, the steady increase in the hydrostatic-to-explosive $[\mathrm{Mg} / \mathrm{Ca}]$ abundance ratio in the innermost, more metal-rich regions of the inner disk suggests a more complex relation between the IMF and the nucleosynthesis of $\mathrm{SNe}$ Ia and SNe II (McWilliam et al. 2013). More recently, Kudritzki et al. (2015) have called attention to the chemical enrichment of star-forming galaxies using analytical chemical evolution models. They find that their sample can be split into three groups according to the rate either of the galactic wind mass loss or of the accretion mass gain to the star formation rate. They applied their model to the Galaxy and find that the actual iron gradient would imply a modest accretion rate and a moderate mass-loading factor. The homogeneity of the above $\alpha$-element gradients can provide new firm constraints on the role that the IMF and the infall rate played in the star formation history of the Galactic thin disk.

Acknowledgements. This work was partially supported by PRIN-MIUR (2010 LY5N2T) "Chemical and dynamical evolution of the Milky Way and Local Group galaxies" (P.I.: F. Matteucci). M.F. acknowledges the financial support from the PO FSE Abruzzo 2007-2013 through the grant "Spectrophotometric characterization of stellar populations in Local Group dwarf galaxies" prot. 89/2014/OACTe/D (P.I.: S. Cassisi). We also acknowledge an anonymous referee for the positive opinions concerning this experiment and for the very pertinent suggestions that improved the content and the readability of the paper.

\section{References}

Anderson, R. I., Ekström, S., Georgy, C., et al. 2014, A\&A, 564, A100 Andrievsky, S. M., Luck, R. E., Martin, P., \& Lépine, J. R. D. 2004, A\&A, 413, 159

Arnett, W. D. 1971, ApJ, 166, 153

Balser, D. S., Rood, R. T., Bania, T. M., \& Anderson, L. D. 2011, ApJ, 738, 27

Beers, T. C., Flynn, K., \& Gebhardt, K. 1990, AJ, 100, 32

Bensby, T., Feltzing, S., \& Lundström, I. 2003, A\&A, 410, 527 (B05)

Bersier, D., Burki, G., \& Kurucz, R. L. 1997, A\&A, 320, 228

Bono, G., Caputo, F., Cassini, S., et al. 2000, ApJ, 543, 955

Bono, G., Marconi, M., Cassini, S., et al. 2005, ApJ, 621, 966

Bragaglia, A., Sestito, P., Villanova, S., et al. 2008, A\&A, 480, 79

Bresolin, F. 2011, ApJ, 729, 56

Calura, F., Recchi, S., Matteucci, F., \& Kroupa, P. 2010, MNRAS, 406, 1985

Carraro, G., de La Fuente Marcos, R., Villanova, S., et al. 2007a, A\&A, 466, 931

Carraro, G., Geisler, D., Villanova, S., Frinchaboy, P. M., \& Majewski, S. R. 2007b, A\&A, 476, 217

Carretta, E., Bragaglia, A., Gratton, R. G., \& Lucatello, S. 2009, A\&A, 505, 139

Carretta, E., Bragaglia, A., Gratton, R. G., et al. 2014, A\&A, 564, A60

Cescutti, G., Matteucci, F., François, P., \& Chiappini, C. 2007, A\&A, 462, 943

Cescutti, G., Matteucci, F., Lanfranchi, G. A., \& McWilliam, A. 2008, A\&A, 491, 401

Chen, L., Hou, J. L., \& Wang, J. J. 2003, AJ, 125, 1397

Chiappini, C., Matteucci, F., \& Padoan, P. 1997, From Quantum Fluctuations to Cosmological Structures, 126, 545

Chiappini, C., Matteucci, F., \& Romano, D. 2001, ApJ, 554, 1044

Colavitti, E., Matteucci, F., \& Murante, G. 2008, A\&A, 483, 401

Costa, R. D. D., Uchida, M. M. M., \& Maciel, W. J. 2004, A\&A, 423, 199

Crowther, P. A. 2007, ARA\&A, 45, 177

Daflon, S., \& Cunha, K. 2004, ApJ, 617, 1115

Davies, B., Origlia, L., Kudritzki, R.-P., et al. 2009a, ApJ, 694, 46 (D09a)

Davies, B., Origlia, L., Kudritzki, R.-P., et al. 2009b, ApJ, 696, 2014 (D09b)

Decressin, T., Meynet, G., Charbonnel, C., Prantzos, N., \& Ekström, S. 2007, A\&A, 464, 1029

Denissenkov, P. A. 1994, A\&A, 287, 113

Edmunds, M. G., \& Roy, J.-R. 1993, MNRAS, 261, L17 
K. Genovali et al.: On the $\alpha$-element gradients of the Galactic thin disk using Cepheids

Evans, C. J., Davies, B., Kudritzki, R.-P., et al. 2011, A\&A, 527, A50 Fabrizio, M., Merle, T., Thévenin, F., et al. 2012, PASP, 124, 519 François, P., Matteucci, F., Cayrel, R., et al. 2004, A\&A, 421, 613 Freudling, W., Romaniello, M., Bramich, D. M., et al. 2013, A\&A, 559, A96 Friel, E. D., Janes, K. A., Tavarez, M., et al. 2002, AJ, 124, 2693 Friel, E. D., Jacobson, H. R., \& Pilachowski, C. A. 2010, AJ, 139, 1942 Fu, J., Hou, J. L., Yin, J., \& Chang, R. X. 2009, ApJ, 696, 668 Genovali, K., Lemasle, B., Bono, G., et al. 2013, A\&A, 554, A132 Genovali, K., Lemasle, B., Bono, G., et al. 2014, A\&A, 566, A37 (G14) Gerin, M., Ruaud, M., Goicoechea, J. R., et al. 2015, A\&A, 573, A30 Grevesse, N., Noels, A., \& Sauval, A. J. 1996, Cosmic Abundances, 99, 117 Groenewegen, M. A. T., Udalski, A., \& Bono, G. 2008, A\&A, 481, 441 Gustafsson, B., Edvardsson, B., Eriksson, K., et al. 2008, A\&A, 486, 951 Harris, H. C. 1985 , AJ, 90, 756

Henry, R. B. C., Kwitter, K. B., \& Balick, B. 2004, AJ, 127, 2284

Henry, R. B. C., Kwitter, K. B., Jaskot, A. E., et al. 2010, ApJ, 724, 748 Inno, L., Matsunaga, N., \& Bono, G. 2013, ApJ, 764, 84

Jacobson, H. R., Friel, E. D., \& Pilachowski, C. A. 2008, AJ, 135, 2341 Jacobson, H. R., Friel, E. D., \& Pilachowski, C. A. 2009, AJ, 137, 4753 Jacobson, H. R., Friel, E. D., \& Pilachowski, C. A. 2011a, AJ, 141, 58 Jacobson, H. R., Pilachowski, C. A., \& Friel, E. D. 2011b, AJ, 142, 59 Johnson, C. I., \& Pilachowski, C. A. 2012, ApJ, 754, L38

Karakas, A. I., García-Hernández, D. A., \& Lugaro, M. 2012, ApJ, 751, 8 Korotin, S. A., Andrievsky, S. M., Luck, R. E., et al. 2014, MNRAS, 444, 3301 Kovtyukh, V. V., \& Gorlova, N. I. 2000, A\&A, 358, 587

Kubryk, M., Prantzos, N., \& Athanassoula, E. 2015a, A\&A, in press, DOI: $10.1051 / 0004-6361 / 201424171$

Kubryk, M., Prantzos, N., \& Athanassoula, E. 2015b, A\&A, in press, DOI: $10.1051 / 0004-6361 / 201424599$

Kudritzki, R.-P., Urbaneja, M. A., Bresolin, F., et al. 2008, ApJ, 681, 269 Kudritzki, R.-P., Urbaneja, M. A., Gazak, Z., et al. 2012, ApJ, 747, A15 Kudritzki, R.-P., Urbaneja, M. A., Bresolin, F., et al. 2014, ApJ, 788, A56 Kudritzki, R.-P., Ho, I.-T., Schruba, A., et al. 2015, MNRAS, 450, 342

Lemasle, B., François, P., Bono, G., et al. 2007, A\&A, 467, 283

Lemasle, B., François, P., Genovali, K., et al. 2013, A\&A, 558, A31 (LEM)

Lépine, J. R. D., Cruz, P., Scarano, S., Jr., et al. 2011, MNRAS, 417, 698

Limongi, M., \& Chieffi, A. 2012, ApJS, 199, 38

Loomis, C., Schmidt, E. G., \& Simon, N. R. 1988, MNRAS, 235, 1059

Luck, R. E., \& Lambert, D. L. 2011, AJ, 142, 136 (LIII)

Luck, R. E., Andrievsky, S. M., Kovtyukh, V. V., Gieren, W., \& Graczyk, D. 2011, AJ, 142, 51 (LII)

Maciel, W. J., \& Quireza, C. 1999, A\&A, 345, 629

Maeder, A. 2009, in Physics, Formation, and Evolution of Rotating Stars, Astron. Astrophys. Lib. (Heidelberg Berlin: Springer)

Magrini, L., Stanghellini, L., \& Villaver, E. 2009, ApJ, 696, 729

Magrini, L., Stanghellini, L., Corbelli, E., et al. 2010a, A\&A, 512, A63

Magrini, L., Randich, S., Zoccali, M., et al. 2010b, A\&A, 523, A11

Marino, R. A., Gil de Paz, A., Castillo-Morales, A., et al. 2012, ApJ, 754, A61

Martin, P., \& Roy, J.-R. 1994, ApJ, 424, 599

Matsunaga, N., Kawadu, T., Nishiyama, S., et al. 2011, Nature, 477, 188

Matsunaga, N., Feast, M. W., Kawadu, T., et al. 2013, MNRAS, 429, 385

Matteucci, F. 2003, Ap\&SS, 284, 539

Matteucci, F., \& François, P. 1989, MNRAS, 239, 885

Matteucci, F., Romano, D., Arcones, A., Korobkin, O., \& Rosswog, S. 2014, MNRAS, 438, 2177

McWilliam, A., Wallerstein, G., \& Mottini, M. 2013, ApJ, 778, 149
Merle, T., Thévenin, F., Pichon, B., \& Bigot, L. 2011, MNRAS, 418, 863 Mikolaitis, Š., Hill, V., Recio-Blanco, A., et al. 2014, A\&A, 572, A33 (M14)

Minchev, I., Chiappini, C., \& Martig, M. 2013, A\&A, 558, A9

Minchev, I., Chiappini, C., \& Martig, M. 2014, A\&A, 572, A92

Mollá, M., \& Díaz, A. I. 2005, MNRAS, 358, 521

Najarro, F., Figer, D. F., Hillier, D. J., Geballe, T. R., \& Kudritzki, R. P. 2009, ApJ, 691, 1816 (N09)

Origlia, L., Oliva, E., Maiolino, R., et al. 2013, A\&A, 560, A46 (O13)

Pancino, E., Carrera, R., Rossetti, E., \& Gallart, C. 2010, A\&A, 511, A56

Perinotto, M., \& Morbidelli, L. 2006, MNRAS, 372, 45

Pilyugin, L. S., Grebel, E. K., \& Kniazev, A. Y. 2014a, AJ, 147, 131

Pilyugin, L. S., Grebel, E. K., Zinchenko, I. A., \& Kniazev, A. Y. 2014b, AJ 148,134

Pottasch, S. R., \& Bernard-Salas, J. 2006, A\&A, 457, 189

Prantzos, N., \& Boissier, S. 2000, MNRAS, 313, 338

Quireza, C., Rood, R. T., Bania, T. M., Balser, D. S., \& Maciel, W. J. 2006, ApJ, 653,1226

Ramírez, S. V., Arendt, R. G., Sellgren, K., et al. 2008, ApJS, 175. 147

Recio-Blanco, A., de Laverny, P., Kordopatis, G., et al. 2014, A\&A, 567, A5

Reddy, B. E., Tomkin, J., Lambert, D. L., \& Prieto, C. A. 2003, MNRAS, 340, 304 (R03)

Rolleston, W. R. J., Smartt, S. J., Dufton, P. L., \& Ryans, R. S. I. 2000, A\&A, 363,537

Rudolph, A. L., Fich, M., Bell, G. R., et al. 2006, ApJS, 162, 346

Sánchez, S. F., Rosales-Ortega, F. F., Iglesias-Páramo, J., et al. 2014, A\&A, 563, A49

Sanders, N., Caldwell, N., \& McDowell, J. 2012, ApJ, 758, 133

Sasselov, D. D. 1986, PASP, 98, 561

Sestito, P., Bragaglia, A., Randich, S., et al. 2006, A\&A, 458, 121

Sestito, P., Randich, S., \& Bragaglia, A. 2007, A\&A, 465, 185

Smartt, S. J., \& Rolleston, W. R. J. 1997, ApJ, 481, L47

Soubiran, C., \& Girard, P. 2005, A\&A, 438, 139 (SG05)

Sousa, S. G., Santos, N. C., Israelian, G., Mayor, M., \& Monteiro, J. P. F. G. 2007, A\&A, 469, 783

Spite, M. 1967, Ann. Astrophys., 30, 211

Spitoni, E., Matteucci, F., Recchi, S., Cescutti, G., \& Pipino, A. 2013a, A\&A, 504, A87

Spitoni, E., Matteucci, F., \& Marcon-Uchida, M. M. 2013b, A\&A, 551, A123

Stanghellini, L., Guerrero, M. A., Cunha, K., Manchado, A., \& Villaver, E. 2006, ApJ, 651, 898

Stanghellini, L., Magrini, L., Casasola, V., et al. 2014, A\&A, 567, A88

Stasinska, G., Peña, M., Bresolin, F., et al. 2013, A\&A, 552, A12

Takeda, Y., \& Takada-Hidai, M. 2000, PASJ, 52, 113

Takeda, Y., Kang, D.-I., Han, I., Lee, B.-C., \& Kim, K.-M. 2013, MNRAS, 432, 769

Tinsley, B. M. 1979, ApJ, 229, 1046

Twarog, B. A., Ashman, K. M., \& Anthony-Twarog, B. J. 1997, AJ, 114, 2556

Urbaneja, M. A., Herrero, A., Bresolin, F., et al. 2005, ApJ, 622, 862

Ventura, P., D’Antona, F., \& Mazzitelli, I. 2002, A\&A, 393, 215

Vila-Costas, M. B., \& Edmunds, M. G. 1992, MNRAS, 259, 121

Woosley, S. E., \& Weaver, T. A. 1995, ApJS, 101, 181

Yong, D., Carney, B. W., \& Teixera de Almeida, M. L. 2005, AJ, 130, 597

Yong, D., Carney, B. W., Teixera de Almeida, M. L., \& Pohl, B. L. 2006, AJ $131,2256(\mathrm{YON})$

Yong, D., Carney, B. W., \& Friel, E. D. 2012, AJ, 144, 95

Zaritsky, D., Kennicutt, R. C., Jr., \& Huchra, J. P. 1994, ApJ, 420, 87

Zurita, A., \& Bresolin, F. 2012, MNRAS, 427, 1463 